TRANSACTIONS OF THE

AMERICAN MATHEMATICAL SOCIETY

Volume 361, Number 5, May 2009, Pages 2277-2304

S 0002-9947(08)04830-7

Article electronically published on December 23, 2008

\title{
GENERALIZED STARK FORMULAE OVER FUNCTION FIELDS
}

\author{
KI-SENG TAN
}

\begin{abstract}
We establish formulae of Stark type for the Stickelberger elements in the function field setting. Our result generalizes work of Hayes and a conjecture of Gross. It is used to deduce a $p$-adic version of the Rubin-Stark Conjecture and the Burns Conjecture.
\end{abstract}

\section{INTRODUCTION}

In this paper, we study Stickelberger elements related to abelian extensions over global function fields. Our main result is Theorem 5.1, which generalizes a theorem of Hayes ([Hay88 $)$. In a way the theorem puts together conjectures of Gross, Rubin and Stark. Also, we will show that it implies a $p$-adic version of the Rubin-Stark Conjecture (see Theorem 1.5 below). Furthermore, using the theorem, we are able to deduce a $p$-adic version of a formula conjectured by Burns (see Theorem 1.7 below).

For the purpose of having a better description of our work, we shall review in the following paragraphs both the Rubin-Stark Conjecture and the Burns Conjecture. But before we do so, let us first fix some notation.

From now on, $K / k$ will be a finite abelian extension over a global function field of characteristic $p$. We assume that the extension is unramified outside a given finite set $S$ of places of $k$. Also, we fix another finite non-empty set $T$ of places of $k$ such that $T \cap S=\emptyset$. The notation $K^{\prime}$ will be used to denote a subfield of $K$ containing $k$. Also, $S\left(K^{\prime}\right)$ (resp. $T\left(K^{\prime}\right)$ ) will denote the set of places of $K^{\prime}$ sitting over $S$ (resp. $T$ ). Let $\mathbb{F}_{q}$ be the constant field of $k$, and put $\Gamma=\operatorname{Gal}(K / k)$, $\Gamma^{\prime}=\operatorname{Gal}\left(K^{\prime} / k\right)$.

The analytical side of the Rubin-Stark Conjecture involves the equivariant $L$ function which interpolates $L$-functions at each character. Recall that for each $\chi \in \hat{\Gamma}$ the modified Artin $L$-function over $k$ is defined as (Gro88])

$$
L_{S, T}(\chi, s)=\prod_{v \in T}\left(1-\chi([v]) \cdot N(v)^{1-s}\right) \prod_{v \notin S}\left(1-\chi([v]) \cdot N(v)^{-s}\right)^{-1} .
$$

Here $[v]$ is the Frobenius element at $v$ and $N(v)=q^{\operatorname{deg}(v)}$ is the norm of $v$. Because in a global field places of the same degree always form a finite set, one can easily

Received by the editors June 26, 2006.

2000 Mathematics Subject Classification. Primary 11S40; Secondary 11R42, 11R58.

Key words and phrases. Stickelberger element, special values of $L$-functions, Stark Conjecture, conjecture of Gross, class numbers, local Leopoldt conjecture, Rubin's conjecture, conjecture of Rubin and Burns, regulators.

The author was supported in part by the National Science Council of Taiwan, NSC91-2115M-002-001, NSC93-2115-M-002-007. 
deduce that the above infinite product is expanded in a unique way as a formal power series in $q^{-s}$. It is well known that this formal power series is in fact a polynomial in $q^{-s}$ ([Tat84]). Applying the theory of Fourier transforms, we see that there is a polynomial $\Theta_{\Gamma, S, T}(s) \in \mathbb{C}[\Gamma]\left[q^{-s}\right]$ such that for every $\chi \in \hat{\Gamma}$

$$
\chi\left(\Theta_{\Gamma, S, T}(s)\right)=L_{S, T}(\chi, s) .
$$

This $\Theta_{\Gamma, S, T}(s)$ is called the modified equivariant $L$-function. From (1), we are able to express $\Theta_{\Gamma, S, T}$ as an infinite product. Namely,

$$
\Theta_{\Gamma, S, T}(s)=\prod_{v \in T}\left(1-[v] \cdot q^{\operatorname{deg}(v)(1-s)}\right) \prod_{v \notin S}\left(1-[v] \cdot q^{-\operatorname{deg}(v) s}\right)^{-1} .
$$

In particular, this implies that $\Theta_{\Gamma, S, T}$ is actually an element in $\mathbb{Z}[\Gamma]\left[q^{-s}\right]$.

Now let us start to describe the arithmetic side of the Rubin-Stark Conjecture. This will involve various regulator maps related to unit groups. Consider $\mathcal{O}_{S\left(K^{\prime}\right)}$, the ring of $S\left(K^{\prime}\right)$-integers of $K^{\prime}$, and let $\mathcal{O}_{S\left(K^{\prime}\right)}^{*}$ be its unit group.

Definition 1.1. Define $U\left(K^{\prime}\right)$ to be the kernel of the reduction modulo $T\left(K^{\prime}\right)$,

$$
\mathcal{O}_{S\left(K^{\prime}\right)}^{*} \longrightarrow \bigoplus_{w \in T\left(K^{\prime}\right)} \mathcal{O}_{w}^{*} /\left(1+\pi_{w} \cdot \mathcal{O}_{w}\right)
$$

and define $r_{K^{\prime}}=\# S\left(K^{\prime}\right)-1$. For simplicity, we denote $U=U(K), r=r_{K}$.

Note that $U\left(K^{\prime}\right)=U^{\operatorname{Gal}\left(K / K^{\prime}\right)}$ is a free abelian group of rank $r_{K^{\prime}}$ and there is an exact sequence (Gro88])

$$
1 \longrightarrow U\left(K^{\prime}\right) \longrightarrow \mathcal{O}_{S\left(K^{\prime}\right)}^{*} \longrightarrow \prod_{w \in T\left(K^{\prime}\right)} \mathbb{F}_{w}^{*} \longrightarrow \operatorname{Pic}\left(\mathcal{O}_{S\left(K^{\prime}\right)}\right)_{T\left(K^{\prime}\right)} \longrightarrow \operatorname{Pic}\left(\mathcal{O}_{S\left(K^{\prime}\right)}\right) \longrightarrow 1
$$

where $\mathbb{F}_{w}^{*}$ is the residue field at $w$. We recall that the $\left(S\left(K^{\prime}\right), T\left(K^{\prime}\right)\right)$-class number of $K^{\prime}$ is the group order

$$
h_{K^{\prime}, S\left(K^{\prime}\right), T\left(K^{\prime}\right)}=\left|\operatorname{Pic}\left(\mathcal{O}_{S\left(K^{\prime}\right)}\right)_{T\left(K^{\prime}\right)}\right| .
$$

To construct the regulator maps, we shall follow the notation and the methods used in Rub96]. In particular, if $M$ is a finitely generated $\mathbb{Z}[\Gamma]$-module, then $\mathbb{Q} M$ denotes $\mathbb{Q} \otimes M$ and the dual module $M^{*}$ is defined as $\operatorname{Hom}_{\Gamma}(M, \mathbb{Z}[\Gamma]) \subset$ $\operatorname{Hom}_{\Gamma}(\mathbb{Q} M, \mathbb{Q}[\Gamma])$. Also, if $n$ is a non-negative integer, then $\Lambda^{n} M$ denotes the $n$th exterior power of $M$ in the category of $\mathbb{Z}[\Gamma]$-modules. We let $\iota$ denote the natural map $\left(\right.$ Rub96], Sec.1.2) $\iota: \Lambda^{n}\left(M^{*}\right) \longrightarrow\left(\Lambda^{n} M\right)^{*}$, such that if $\phi_{1}, \ldots \phi_{n} \in M^{*}$ and $m_{1}, \ldots, m_{n} \in M$, then

$$
\iota\left(\phi_{1} \wedge \cdots \wedge \phi_{n}\right)\left(m_{1} \wedge \cdots \wedge m_{n}\right)=\operatorname{det}\left(\phi_{i}\left(m_{j}\right)\right) .
$$

Also, following [Rub96], we define

$$
\Lambda_{0}^{n} M:=\left\{m \in \mathbb{Q} \Lambda^{n} M \mid \iota\left(\phi_{1} \wedge \cdots \wedge \phi_{n}\right)(m) \in \mathbb{Z}[\Gamma] \text { for every } \phi_{1}, \ldots, \phi_{n} \in M^{*}\right\} .
$$

Now, we start to define the regulator maps. First, let $Y\left(K^{\prime}\right)=\bigoplus_{w \in S\left(K^{\prime}\right)} \mathbb{Z} \cdot w$ and

$$
X\left(K^{\prime}\right)=\left\{\sum_{w \in S\left(K^{\prime}\right)} a_{w} \cdot w \in Y\left(K^{\prime}\right) \mid \sum_{w \in S\left(K^{\prime}\right)} a_{w}=0\right\} .
$$

For each place $w$ of $K$, let $\operatorname{deg}_{w}$ be the local degree map

$$
\operatorname{deg}_{w}: K_{w}^{*} \longrightarrow K_{w}^{*} / \mathcal{O}_{w}^{*} \longrightarrow \mathbb{Z}
$$


such that if ||$_{w}$ is the normalized absolute value associated to $w$, then

$$
\log \left(|x|_{w}\right)=-\operatorname{deg}_{w}(x) \cdot \log (q) .
$$

Compose this local degree map with the natural embedding $U \longrightarrow K_{w}^{*}$ to form

$$
\lambda_{w}: U \longrightarrow K_{w}^{*} \stackrel{\operatorname{deg}_{w}}{\longrightarrow} \mathbb{Z} .
$$

Also, we define $\lambda: U(K) \longrightarrow X(K)$ to be the $\Gamma$-equivariant homomorphism such that $\lambda(u)=\sum_{w \in S(K)} \lambda_{w}(u) \cdot w$ for every $u \in U(K)$. Write $\lambda^{(n)}: \Lambda^{n} U \longrightarrow \Lambda^{n} X(K)$ (resp. $\left.i^{(n)}: \Lambda^{n} X(K) \stackrel{i^{(n)}}{\longrightarrow} \Lambda^{n} Y(K)\right)$ for the map induced by the map $\lambda$ (resp. the inclusion $i: X(K) \longrightarrow Y(K))$. Then the regulator map

$$
\mathcal{R}_{\Psi}: \mathbb{Q} \Lambda^{n} U \longrightarrow \mathbb{Q}[\Gamma]
$$

associated to a $\Psi \in \Lambda^{n} Y(K)^{*}$ is defined as the one that linearly extends the map

$$
\Lambda^{n} U \stackrel{\lambda^{(n)}}{\longrightarrow} \Lambda^{n} X(K) \stackrel{i^{(n)}}{\longrightarrow} \Lambda^{n} Y(K) \stackrel{\iota(\Psi)}{\longrightarrow} \mathbb{Z}[\Gamma] .
$$

It is easy to see that if $\psi_{i} \in Y(K)^{*}$, then $\mathcal{R}_{\psi_{i}} \in U^{*}$. Also, if $\Psi=\psi_{1} \wedge \cdots \wedge \psi_{n}$, then we have

$$
\mathcal{R}_{\Psi}=\iota\left(\mathcal{R}_{\psi_{1}} \wedge \cdots \wedge \mathcal{R}_{\psi_{n}}\right) .
$$

Consequently, we have $\mathcal{R}_{\Psi}\left(\Lambda_{0}^{n} U\right) \subset \mathbb{Z}[\Gamma]$ for every $\Psi \in \Lambda^{n} Y(K)^{*}$. In this paper, some special elements in $Y(K)^{*}$ of the form $w^{*}$ will be used. Recall that for each place $w$ over $K$, the element $w^{*} \in Y(K)^{*}$ is defined ([Rub96]) such that for every place $w^{\prime}$

$$
w^{*}\left(w^{\prime}\right)=\sum_{\gamma w=w^{\prime}} \gamma
$$

What we call the Rubin-Stark Conjecture is the one proposed by Rubin in Rub96 (Conjecture B'), because it can be viewed as an integral version of Stark's conjecture ( $\underline{\text { San87, }}$, Stk71, Stk75, Stk76, Stk80]). In a way, the conjecture relates some derivative of the equivariant $L$-function to a certain exterior product of units arising from regular representations of $\Gamma$. It is easy to see that the unit group $U$ contains a regular representation of $\Gamma$ if and only if some place in $S$ splits completely over $K$. Thus, for the purpose of having an interesting theory, we need to assume the following:

Assumption 1.2. From now on, we assume that there exist $n, n \geq 1$, different places $S_{0}=\left\{v_{1}, \ldots, v_{n}\right\} \subsetneq S$ such that every place in $S_{0}$ splits completely over $K$.

Definition 1.3. Let $v_{1}, \ldots, v_{n}$ be as in Assumption 1.2 and let $w_{1}, \ldots, w_{n}$ be a fixed set of places of $K$ such that each $w_{i}$ is sitting over the place $v_{i} \in S$. Also, let $\eta=w_{1}^{*} \wedge \cdots \wedge w_{n}^{*}$.

First we note that Assumption 1.2 (together with the class number formula at $s=0)$ implies

$$
\Theta_{\Gamma, S, T}=a_{n}\left(q^{-s}-1\right)^{n}+\cdots \in\left(q^{-s}-1\right) \cdot \mathbb{Z}[\Gamma]\left[q^{-s}\right] .
$$

Also, the coefficient $a_{n} \in \mathbb{Z}[\Gamma]$ will be denoted as $\Theta_{\Gamma, S, T}^{(n)}(0)$. For each $\chi \in \hat{\Gamma}$ let $e_{\chi}$ be the associated idempotent element in the group ring $\mathbb{C}[\Gamma]$, and let $r_{\chi}$ denote the $\mathbb{C}$-dimension of the $\chi$-eigenspace of $\mathbb{C} \otimes_{\mathbb{Z}} U$. Define

$$
\Lambda_{S, T}^{n}=\left\{u \in \Lambda_{0}^{n} U \mid e_{\chi}(u)=0 \text {, for every } \chi \in \hat{\Gamma} \text { such that } r_{\chi}>n\right\} .
$$

Then, in our setting, the Rubin-Stark Conjecture reads as follows. 
Conjecture 1.4 (Rubin, Rub96, Conjecture $\mathrm{B}^{\prime}$ ). There exists an $\epsilon \in \Lambda_{S, T}^{n}$ such that

$$
\mathcal{R}_{\eta}(\epsilon)=\Theta_{\Gamma, S, T}^{(n)}(0) .
$$

We will show in Section 5.1 that our main result implies the following $p$-adic version of the conjecture. Here " $p$-adic" means tensoring things with $\mathbb{Z}_{(p)}$. In particular,

$$
\mathbb{Z}_{(p)} \Lambda_{S, T}=\mathbb{Z}_{(p)} \otimes \Lambda_{S, T} \subset \mathbb{Q} \Lambda_{S, T} .
$$

Theorem 1.5. There exists an $\epsilon \in \mathbb{Z}_{(p)} \Lambda_{S, T}^{n}$ such that in $\mathbb{Z}_{(p)}[\Gamma]$

$$
\mathcal{R}_{\eta}(\epsilon)=\Theta_{\Gamma, S, T}^{(n)}(0) .
$$

A different proof of the theorem can be found in Pop05, and a proof for the $l$-adic $(l \neq p)$ version of the Rubin-Stark Conjecture is given in [Bun07]. In view of this, over function fields, the Rubin-Stark Conjecture actually holds. Now we review the conjecture of Burns. We will follow the construction described in Bun02, Haw04. The conjecture involves regulators of another type, and we are going to define them in the follow paragraphs.

First we note that if $M$ is a $\mathbb{Z}[\Gamma]$-module, then for each $\phi \in M^{*}$ there is a unique $\phi^{(i d)} \in \operatorname{Hom}_{\mathbb{Z}}(M, \mathbb{Z})$ such that for $x \in M$

$$
\phi(x)=\sum_{\gamma \in \Gamma} \phi^{(i d)}\left(\gamma^{-1} x\right) \gamma .
$$

For a place $v$ over $k$, define

$$
\bar{\lambda}_{v, \Gamma}: U(k) \longrightarrow k_{v}^{*} \longrightarrow \Gamma_{v} \hookrightarrow \Gamma,
$$

where the first and the last arrows are natural embeddings and the second is the norm residue map in local class field theory. Let $u_{1}, \ldots, u_{r_{k}}$ be a $\mathbb{Z}$-basis for $U(k)$, $v_{n+1}, \ldots, v_{r_{k}}$ be distinct places in $S \backslash S_{0}$ and $\phi_{1}, \ldots, \phi_{n} \in U^{*}$. Consider the matrix $A=\left(a_{i j}\right)_{1 \leq i, j \leq r_{k}}$ with

$$
a_{i j}=\left\{\begin{array}{l}
\phi_{i}^{(i d)}\left(u_{j}\right), \text { if } 1 \leq i \leq n, \\
\bar{\lambda}_{v_{i}, \Gamma}\left(u_{j}\right)-1, \text { if } n+1 \leq i \leq r_{k} .
\end{array}\right.
$$

For each pair $i, j$ the entry $a_{i j}$ is an element in $\mathbb{Z}[\Gamma]$, and it is obvious that the determinant $\operatorname{det}(A)$ is in $I^{r_{k}-n}$ where $I$ is the augmentation ideal of $\mathbb{Z}[\Gamma]$. Up to \pm 1 , the residue class of $\operatorname{det}(A)$ modulo $I^{r_{k}-n+1}$ depends on neither the ordering of $v_{1}, \ldots, v_{r_{k}}$ nor the choice of the basis $u_{1}, \ldots, u_{r_{k}}$. We assume that the ordering of $v_{1}, \ldots, v_{r_{k}}$ is fixed and the basis $u_{1}, \ldots, u_{r_{k}}$ is ordered in a way such that the classical regulator formed by them is positive. On the other hand, the residue class of $\operatorname{det}(A)$ actually depends on the exterior product $\Phi=\iota\left(\phi_{1} \wedge \cdots \wedge \phi_{n}\right) \in \iota\left(\Lambda^{n} U^{*}\right)$, and therefore we will denote it as $\operatorname{Reg}_{\Gamma}^{\Phi}$. On top of Conjecture 1.4, Burns Bun02] proposes the following strengthened conjecture.

Conjecture 1.6. Assume that Conjecture 1.4 holds, so that for every $\Phi \in \iota\left(\Lambda^{n} U^{*}\right)$, we have $\Phi(\epsilon) \in \mathbb{Z}[\Gamma]$. Then this element satisfies

$$
\Phi(\epsilon) \equiv h_{k, S, T} \operatorname{Reg}_{\Gamma}^{\Phi} \quad\left(\bmod I^{r_{k}-n+1}\right) .
$$


For more material related to this conjecture, see for instance Bun02, Bun07, Haw04, Pop99a, Pop99b, Pop02, Rub96]. The $l$-adic version (for $l \neq p$ ) of the conjecture is proved in [Bun07, but it seems the technique used in the proof cannot be applied to cover the following $p$-adic version, which will be proved in Section 5.2. Let $I_{p}$ be the augmentation ideal of $\mathbb{Z}_{p}[\Gamma]$.

Theorem 1.7. Let the notation be as in Theorem[1.5. Then for every $\Phi \in \iota\left(\Lambda^{n} U^{*}\right)$, we have $\Phi(\epsilon) \in \mathbb{Z}_{p}[\Gamma]$, and this element satisfies

$$
\Phi(\epsilon) \equiv h_{k, S, T} \operatorname{Reg}_{\Gamma}^{\Phi} \quad\left(\bmod I_{p}^{r_{k}-n+1}\right) .
$$

Now we begin to describe Theorem 5.1, our main result. In short, it is a $p$-adic refinement of Theorem 1.5. The method for making this kind of refinement comes from [Gro88, Gro90], and the main idea is to replace $\mathbb{Z}_{p}$ by certain Galois groups in order to construct refinements of both side of the equality (9). To explain it, let us start with those degree maps $\operatorname{deg}_{w}$ which play important roles in the construction of the regulator maps. These local degree maps together form the global degree map deg : $\mathbb{A}_{K}^{*} \longrightarrow \mathbb{Z}$ defined on the idèle group $\mathbb{A}_{K}^{*}$. Let $L_{0}=K \mathbb{F}_{q^{p}}$ be the constant $\mathbb{Z}_{p}$-extension over $K$. If we view $\mathbb{Z}_{p}$ as the Galois group $\operatorname{Gal}\left(L_{0} / K\right)$ and compose the map deg with the embedding $\mathbb{Z} \longrightarrow \mathbb{Z}_{p}$ which sends 1 to the Frobenius in $\operatorname{Gal}\left(L_{0} / K\right)$, then we get the norm residue map $\mathbb{A}_{K}^{*} \longrightarrow \operatorname{Gal}\left(L_{0} / K\right)$, and the local degree map is just the composite $K_{w}^{*} \longrightarrow \mathbb{A}_{K}^{*} \longrightarrow \operatorname{Gal}\left(L_{0} / K\right)$. From this we see that the field extension $L_{0} / K$ and the related norm residue maps are implicitly used in the construction of the previous regulator maps.

For the refinements we are going to use various Galois groups of the form $H:=$ $\operatorname{Gal}(L / K)$, where $L / K$ is a pro- $p$ abelian extension such that $L / k$ is also abelian and unramified outside $S$ (such extension is called admissible, see Definition 2.1). We let $H$ play the role of $\mathbb{Z}_{p}=\operatorname{Gal}\left(L_{0} / K\right)$ and use the related norm residue maps to construct, for each $\Psi$, the associated refined regulator map $\mathcal{R}_{\Psi, H}$ (Definition 4.2) which has values in the $n$th relative augmentation quotient associated to $H$ (Definition 2.6). To see that $\mathcal{R}_{\Psi, H}$ actually refines $\mathcal{R}_{\Psi}$, we only need to take $L=L_{0}$, because in this situation $\mathcal{R}_{\Psi}$ can be recovered from $\mathcal{R}_{\Psi, H}$ (Lemma 4.4). We would like to emphasize that $\mathbb{H}$, the Galois group of the maximal admissible extension, is a direct product of countable infinite many copies of $\mathbb{Z}_{p}$ (Lemma 2.2). Also, in a way, Lemma 2.10 together with the isomorphism (15) says that an element in the $n$th relative augmentation quotient associated to $\mathbb{H}$ can be identified as a $\mathbb{Z}_{p}[\Gamma]$-coefficient $n$th degree homogeneous "polynomial in countable infinite many variables". Furthermore, under this identification, if the $\mathbb{Z}_{p}$-basis of $\mathbb{H}$ is suitably arranged, then for each $\epsilon \in \mathbb{Z}_{(p)} \Lambda_{S, T}^{(n)}$ the value $\mathcal{R}_{\Psi}(\epsilon)$ is just the coefficient of a certain monomial in $\mathcal{R}_{\Psi, \mathbb{H}}(\epsilon)$. In particular, it is fair to say that the map $\mathcal{R}_{\eta, \mathbb{H}}$, where $\eta$ is the element in Definition 1.3. carries a rich amount of information about the unit group. In fact, the universal property studied in Section 4.4 (see Corollary 4.10) tells us that most of the important information about the integer structure of the $n$th exterior product of the units group can be obtained from $\mathcal{R}_{\eta, \mathbb{H}}$.

The refinement of the equivariant $L$-function $\Theta_{\Gamma, S, T}$ turns out to be the Stickelberger element $\theta_{G}$ (see Definition 3.1) where $G=\operatorname{Gal}(L / k)$. For its reason, please see Lemma 3.2. It is somewhat surprising, since the Stickelberger element only interpolates special values of $L$-functions while the equivariant $L$-function interpolates the complete $L$-functions. Lemma 3.2 also tells us that in the case where $L=L_{0}$, the " $n$th derivative" $\Theta_{\Gamma, S, T}^{(n)}(0)$ can be recovered from the residue class 
$\left[\theta_{G}\right]_{(n, H)}$ of $\theta_{G}$ in the $n$th relative augmentation quotient. That there is a unique $\epsilon$ in $\mathbb{Z}_{(p)} \Lambda_{S, T}^{(n)}$ such that

$$
\left[\theta_{G}\right]_{(n, H)}=\mathcal{R}_{\eta, H}(\epsilon)
$$

for every admissible $H$ is exactly the content of Theorem 5.1. In the case where $H=\mathbb{H}$ we have an equality between two "polynomials in infinitely many variables", while (9) in Theorem 1.5 is an equality between the corresponding coefficients of certain "monomials". This is the reason why Theorem 5.1 implies Theorem 1.5

The proof of Theorem 1.7 involves a refined class number formula proposed by Gross Gro88 (see Conjecture 5.2). What we actually use is its $p$-adic version proved in Tan95, (see Theorem 5.3) in which the congruence (38) relates the Stickelberger element $\theta_{G}$ with the product of $h_{k, S, T}$ and a regulator $\operatorname{det}_{G}$ defined by Gross. In contrast to this, Theorem 5.1 relates $\theta_{G}$ with the refined regulator $\mathcal{R}_{\eta, H}(\epsilon)$ which is the left-hand side of the congruence (12). The main step for proving Theorem 1.7 is to use the aforementioned universal property to relate the right-hand side of (12) to the product $h_{k, S, T} \operatorname{det}_{G}$.

Finally, let us have some words about the proof of Theorem [5.1. In brief, it is based on two observations. First, we find that, via Fourier transform, the theorem is equivalent to its twisted version, Theorem [5.4, in which the main part is the congruence (43). Also, we have discovered that both the left-hand and right-hand sides of (43) can be found as factors of the corresponding left-hand and right-hand sides of the congruence (38) in Theorem 5.3. Furthermore, the two sides of (38) are indeed products of these kind of factors (indexed by characters; see Proposition 6.15 and Proposition (7.1). To use (38) to prove (43), we apply Fourier transforms, the universal property and the result of Hayes for the case $n=1$.

This manuscript has evolved through several versions since 1996. It is a great pleasure to thank David Burns, Wen-Cheng Chi, Benedict Gross, Po-Yi Huang, Ka-Lam Kueh, King F. Lai, Cristian Popescu, Karl Rubin and John Tate for stimulating discussions.

\section{Admissible extensions and augmentation quotients}

In this chapter, we study admissible extensions and the properties of the associated augmentation quotients.

\subsection{Admissible extensions.}

Definition 2.1. An abelian extension $L / K$ and its Galois group $H=\operatorname{Gal}(L / K)$ are admissible if the following are satisfied:

(1) The extension $L / k$ is abelian and is unramified outside $S$.

(2) The extension $L / K$ is a pro- $p$ extension.

Throughout this paper, we will fix an admissible extension $L / K$, and we will also fix the notation: $G=\operatorname{Gal}(L / k), H=\operatorname{Gal}(L / K)$, and $\Gamma=G / H$. Also, a subgroup of $G$ denoted as $H^{\prime}$ always contains $H$, and we always denote $K^{\prime}=L^{H^{\prime}}$ and $\Gamma^{\prime}=G / H^{\prime}$.

2.2. The maximal admissible extension. Although there are infinitely many different admissible extensions, the theory in this paper can be summed up to a theory for a single extension, that is, the maximal admissible extension with respect to $K / k$ and $S$. We will denote the associated Galois group by $\mathbb{H}$ and will first study its structure. 
Lemma 2.2. The maximal admissible Galois group $\mathbb{H}$ is a direct product of countable infinite many copies of $\mathbb{Z}_{p}$.

Before we prove the lemma, let us recall some known results related to the local Leopoldt conjecture (see Kis93, Tan95).

Lemma 2.3. Suppose that $\mathcal{K}$ is a global function field of characteristic $p$ and $v$ is a place of $\mathcal{K}$. If an element $u \in \mathcal{K}^{*}$ is divisible by $p$ in $\mathcal{K}_{v}^{*}$, then it is divisible by $p$ in $\mathcal{K}^{*}$.

As a consequence, we have the following.

Lemma 2.4 (Kis93]). Suppose that $\mathcal{K}$ is a global function field of characteristic $p$ and $\mathcal{S}$ is a finite set of places of $\mathcal{K}$. Then the Galois group of the maximal pro$p$ abelian extension over $\mathcal{K}$ unramified outside $\mathcal{S}$ is a direct product of countable infinite many copies of $\mathbb{Z}_{p}$.

Proof of Lemma 2.2, Let $\Gamma=\Gamma_{p} \oplus \Gamma_{0}$ be the natural decomposition of $\Gamma$ into the $p$-part, $\Gamma_{p}$, and the non-p-part, $\Gamma_{0}$. Suppose that $\mathbb{G}$ is the Galois group over $k$ of the maximal pro- $p$ abelian extension unramified outside $S$. Then $\mathbb{G}$ is an extension of $\Gamma_{p}$ which is viewed as a quotient group of $\Gamma$. Let $\mathcal{H}=\operatorname{ker}\left(\mathbb{G} \longrightarrow \Gamma_{p}\right)$ be the kernel of the natural quotient map. Then $\mathcal{H}$ is isomorphic to $\mathbb{H}$.

By Lemma 2.4, $\mathbb{G}$ is a direct product of countable infinite many copies of $\mathbb{Z}_{p}$, and so is $\mathbb{H}$.

2.3. Group rings and augmentation ideals. For the rest of this chapter, we will study group rings with various coefficient rings together with two types of augmentation ideals and the associated augmentation quotients.

Let $R$ be an integral domain finite over $\mathbb{Z}$ or $\mathbb{Z}_{p}$. If $C$ is the fraction field of $R$ and $M$ is an $R$-module, then we use $C M$ to denote $C \otimes_{R} M$.

Definition 2.5. For a pro-finite group $\mathcal{H}$, let $R[\mathcal{H}]$ be the projective limit of $R[\Delta]$, where $\Delta$ runs through all the finite quotient groups of $\mathcal{H}$. Also, for every positive integer $n$, let $I_{R}(\mathcal{H})^{n}$ be the projective limit of $I_{R}(\Delta)^{n}$, where $I_{R}(\Delta)^{n}$ is the $n$th power of the augmentation ideal $I_{R}(\Delta)$. We call respectively $I_{R}(\mathcal{H})^{n}$ and $I_{R}(\mathcal{H})^{n} / I_{R}(\mathcal{H})^{n+1}$ the $n$th augmentation ideal and the $n$th augmentation quotient of $R[\mathcal{H}]$.

For the rest of the paper, if $\Xi: \mathcal{H}_{1} \longrightarrow \mathcal{H}_{2}$ is a group homomorphism, then we will also use $\Xi$ to denote the induced homomorphisms on the group rings and the augmentation quotients.

Definition 2.6. If $H^{\prime}$ is finite, let $I_{R, H^{\prime}}$ be the kernel of the ring homomorphism $R[G] \longrightarrow R\left[\Gamma^{\prime}\right]$ induced from the natural quotient map $G \longrightarrow \Gamma^{\prime}$. In general, for every positive integer $n$, let $I_{R, H^{\prime}}^{n}$ be the projective limit of $I_{R, H^{\prime} / \mathcal{N}}^{n}$, where $\mathcal{N}$ runs through the family of all open subgroups of $H^{\prime}$ contained in $H$. We call respectively $I_{R, H^{\prime}}^{n}$ and $I_{R, H^{\prime}}^{n} / I_{R, H^{\prime}}^{n+1}$ the $n$th relative $H^{\prime}$-augmentation ideal and the $n$th relative $H^{\prime}$-augmentation quotient of $R[G]$

For simplicity, we let $I\left(H^{\prime}\right), I_{p}\left(H^{\prime}\right), I_{H^{\prime}}$ and $I_{p, H^{\prime}}$ denote respectively $I_{\mathbb{Z}}\left(H^{\prime}\right)$, $I_{\mathbb{Z}_{p}}\left(H^{\prime}\right), I_{\mathbb{Z}, H^{\prime}}$ and $I_{\mathbb{Z}_{p}, H^{\prime}}$.

Definition 2.7. For $\xi \in I_{R}\left(H^{\prime}\right)^{n} \subset R\left[H^{\prime}\right]$, let $[\xi]_{(n)}$ be its residue class in the augmentation quotient $I_{R}\left(H^{\prime}\right)^{n} / I_{R}\left(H^{\prime}\right)^{n+1}$. Also, for $\xi \in I_{R, H^{\prime}}^{n} \subset R[G]$, let $[\xi]_{\left(n, H^{\prime}\right)}$ be its residue class in $I_{R, H^{\prime}}^{n} / I_{R, H^{\prime}}^{n+1}$. 
Definition 2.8. Let $F$ be a fixed number field containing all the values of characters of $\Gamma$ and let $\mathcal{O}=\mathcal{O}_{F}$ be its ring of integers. Let $\mathcal{O}_{p}$ be the completion of $\mathcal{O}$ at a fixed place sitting over $p$ and let $F_{p}$ be its field of fractions. Define the group rings over $F$, $F_{p}$ and the corresponding augmentation ideals as follows. Let $F\left[H^{\prime}\right]=F \mathcal{O}\left[H^{\prime}\right]$ and $F_{p}\left[H^{\prime}\right]=F_{p} \mathcal{O}_{p}\left[H^{\prime}\right]$. Also, for every positive integer $n$, let $I_{F}\left(H^{\prime}\right)^{n}=F I_{\mathcal{O}}\left(H^{\prime}\right)^{n}$, $I_{F_{p}}\left(H^{\prime}\right)^{n}=F_{p} I_{\mathcal{O}_{p}}\left(H^{\prime}\right)^{n}, I_{F, H^{\prime}}^{n}=F I_{\mathcal{O}, H^{\prime}}^{n}$, and $I_{F_{p}, H^{\prime}}^{n}=F_{p} I_{\mathcal{O}_{p}, H^{\prime}}^{n}$.

In many situations, the structure of the augmentation quotients can be explicitly expressed. First of all, we have the isomorphism

$$
\delta_{H^{\prime}}: H^{\prime} \longrightarrow I\left(H^{\prime}\right) / I\left(H^{\prime}\right)^{2} \text {, }
$$

which sends $h \in H^{\prime}$ to $h-1\left(\bmod I\left(H^{\prime}\right)^{2}\right)$. Also, if $H \simeq \mathbb{Z}_{p}^{d}$ for some $d$ and $R$ is either $\mathbb{Z}_{p}$ or $\mathcal{O}_{p}$, then the graded ring formed by augmentation quotients can be identified with a polynomial ring. To see this, let $R\left[\left[s_{1}, \ldots, s_{d}\right]\right]$ be the ring of formal power series in $d$ variables. If $\mathcal{E}=\left\{\sigma_{1}, \ldots, \sigma_{d}\right\}$ is a basis of $H$ over $\mathbb{Z}_{p}$ and $x_{i}=\sigma_{i}-1 \in R[H], i=1, \ldots, d$, then the map $R[H] \longrightarrow R\left[\left[s_{1}, \ldots, s_{d}\right]\right], x_{i} \mapsto s_{i}$, is an isomorphism. Consequently, for every positive integer $n$,

$$
I_{R}(H)^{n}=\left(x_{1}, \ldots, x_{d}\right)^{n} \simeq\left(s_{1}, \ldots, s_{d}\right)^{n},
$$

and the augmentation quotient $I_{R}(H)^{n} / I_{R}(H)^{n+1}$ is isomorphic to the $R$-module of $n$th degree homogeneous polynomials in $s_{1}, \ldots, s_{d}$. This induces an isomorphism

$$
d_{\mathcal{E}, R}: \bigoplus_{n=0}^{\infty} I_{R}(H)^{n} / I_{R}(H)^{n+1} \longrightarrow R\left[s_{1}, \ldots, s_{d}\right] .
$$

Since $F_{p}[H]=F_{p} \otimes_{\mathcal{O}_{p}} \mathcal{O}_{p}[H]$, tensoring with $F_{p}$, we get the induced ring homomorphism

$$
d_{\mathcal{E}, F_{p}}: \bigoplus_{n=0}^{\infty} I_{F_{p}}(H)^{n} / I_{F_{p}}(H)^{n+1} \longrightarrow F_{p}\left[s_{1}, \ldots, s_{d}\right] .
$$

Lemma 2.9. (a) For each non-negative integer $n$ we have $I_{p}(H)^{n} \cap \mathbb{Z}[H]=$ $I(H)^{n}, I_{\mathcal{O}_{p}}(H)^{n} \cap \mathcal{O}[H]=I_{\mathcal{O}}(H)^{n}$ and $I_{F_{p}}(H)^{n} \cap F[H]=I_{F}(H)^{n}$.

(b) Suppose that either $H$ is finite free over $\mathbb{Z}_{p}$ or $H=\mathbb{H}$. For $i=1,2$, let $A_{i}$ be one of the rings $\mathbb{Z}, \mathbb{Z}_{p}, \mathcal{O}, \mathcal{O}_{p}, F$ and $F_{p}$. If $A_{1} \subset A_{2}$, then for each $n$, $I_{A_{2}}(H)^{n} \cap A_{1}[H]=I_{A_{1}}(H)^{n}$.

(c) If $H$ is finite free over $\mathbb{Z}_{p}$ or $H=\mathbb{H}$, then the natural map

$$
i: I(H)^{n} / I(H)^{n} \longrightarrow I_{p}(H)^{n} / I_{p}(H)^{n+1}
$$

is an isomorphism.

Proof. In part (a), the third equality is from the second. If $H$ is finite, then the proof of the first equality can be found in Tan95, Lemma 2.5. The second equality can be proved in a similar way. If $H=\mathbb{H}$, we prove them by taking projective limits.

To prove part (b), we first assume that $H$ is finite free over $\mathbb{Z}_{p}$. If we are in the special case where $\left\{A_{1}, A_{2}\right\} \subset\left\{\mathbb{Z}_{p}, \mathcal{O}_{p}, F_{p}\right\}$, then part (b) is proved by using (14). In general, put $A_{i}^{\prime}=\left(A_{i}\right)_{p}$, for $i=1,2$. Then we have $I_{A_{2}^{\prime}}(H)^{n} \cap A_{1}^{\prime}[H]=I_{A_{1}^{\prime}}(H)^{n}$. Also, part (a) implies that $I_{A_{i}^{\prime}}(H)^{n} \cap A_{i}[H]=I_{A_{i}}(H)^{n}$. These imply part (b). The case $H=\mathbb{H}$ is proved by taking projective limits.

To prove part (c), we note that by part (b), the map $i$ is injective. First assume that $H$ is finite free over $\mathbb{Z}_{p}$. Then by equation (14), the homomorphism $\phi$ sending 
$h$ to the residue class of $h-1$ is an isomorphism from $H$ to $I_{p}(H) / I_{p}(H)^{2}$. It is obvious that (see (13)) $\phi=i \circ \delta_{H}$. Since $\delta_{H}$ is an isomorphism, so is $i$. This proves the lemma for $n=1$. For $n>1$, we observe that the multiplication map

$$
I_{p} / I_{p}^{2} \times I_{p}^{n-1} / I_{p}^{n} \longrightarrow I_{p}^{n} / I_{P}^{n+1}
$$

may not be surjective but its image generates the whole group (as an abelian group). Then the surjectivity of $i$ is proved by induction. Again, the $H=\mathbb{H}$ case can be proved by taking projective limits.

Lemma 2.10. For $g \in G$, let $\gamma_{g} \in \Gamma$ be its residue class modulo $H$. Then for any non-negative $n$, the homomorphism

$$
£_{n}: R[\Gamma] \otimes_{R} I_{R}(H)^{n} / I_{R}(H)^{n+1} \longrightarrow I_{R, H}^{n} / I_{R, H}^{n+1},
$$

which sends the residue class of $\gamma_{g} \otimes\left(h_{1}-1\right) \cdots\left(h_{n}-1\right)$ to that of $g\left(h_{1}-1\right)$. $\cdots\left(h_{n}-1\right), h_{1}, \ldots, h_{n} \in H$, is an isomorphism.

Proof. That the homomorphism $£_{n}$ is well defined is due to the simple fact that if $h_{1}, h_{2} \in H, g \in G$, then $g h_{1} \equiv g h_{2}\left(\bmod I_{H}\right)$. The rest is obvious for the case where $H$ is finite. In general, it is proved by taking projective limits.

The lemma shows that the structure of the augmentation quotient $I_{R, H}^{n} / I_{R, H}^{n+1}$ actually depends only on the structures of $\Gamma$ and $H$.

2.4. Numerical extensions. The relative $H$-augmentation quotients can be easily expressed in the following situation.

Definition 2.11. The extension $L / K$ and its Galois group $H$ are called numerical if $H \simeq \mathbb{Z}_{p}$.

In particular, $L / K$ is numerical if it is the constant $\mathbb{Z}_{p}$-extension. If $H$ is numerical and $\sigma$ is a $\mathbb{Z}_{p}$-generator of it, then the isomorphisms in the previous sections together form the following isomorphism:

$$
\mathrm{Val}_{\sigma, n}=\operatorname{Val}_{\sigma, n, G / H}: I_{H}^{n} / I_{H}^{n+1} \stackrel{£_{n}^{-1}}{\longrightarrow} \mathbb{Z}[\Gamma] \otimes I(H)^{n} / I(H)^{n+1} \stackrel{d_{\sigma, \mathbb{Z}_{p}}}{\longrightarrow} \mathbb{Z}_{p}[\Gamma] .
$$

Here we identify $\mathbb{Z}[\Gamma] \otimes I(H)^{n} / I(H)^{n+1}$ with $\mathbb{Z}[\Gamma] \otimes I_{p}(H)^{n} / I_{p}(H)^{n+1}$, and we identify a one variable homogeneous polynomial with its coefficient. If $\sigma^{\prime}=u \sigma$, $u \in \mathbb{Z}_{p}^{*}$, is another generator, then $\mathrm{Val}_{\sigma, n}=u^{n} \cdot \mathrm{Val}_{\sigma^{\prime}, n}$.

If $H$ is numerical, then $G$ can be identified with $\Gamma^{\prime} \times H^{\prime}$ for some subgroup $H^{\prime} \simeq \mathbb{Z}_{p}$ containing $H$. In this case, $\Gamma=\Gamma^{\prime} \times H^{\prime} / H$. We will relate the group ring and the $H$-augmentation quotients of the group $G$ to those of the direct product $\breve{G}=\Gamma \times H$. To do so, we let $\varpi=\left|H^{\prime} / H\right|$ and define

$$
\begin{aligned}
¥: G=\Gamma^{\prime} \times H^{\prime} & \longrightarrow \Gamma^{\prime} \times H^{\prime} / H \times H=\Gamma \times H, \\
\left(\gamma^{\prime}, h^{\prime}\right) & \mapsto\left(\gamma^{\prime}, \bar{h}^{\prime}, \varpi h^{\prime}\right) .
\end{aligned}
$$

Both $G$ and $\breve{G}$ are extensions of $\Gamma$ by $H$, and by Lemma 2.10 we have the associated isomorphisms $£_{n, G}: \mathbb{Z}[\Gamma] \otimes I(H)^{n} / I(H)^{n+1} \longrightarrow I_{H}^{n} / I_{H}^{n+1}$ and $£_{n, \breve{G}}: \mathbb{Z}[\Gamma] \otimes$ $I(H)^{n} / I(H)^{n+1} \longrightarrow \breve{I}_{H}^{n} / \breve{I}_{H}^{n+1}$, where $\breve{I}_{H}^{n}$ denotes the $n$th relative $H$-augmentation ideal of $\mathbb{Z}[\breve{G}]$. The following lemma is obvious. 
Lemma 2.12. Let $n$ be a non-negative integer and let $L / K$ be numerical. An element $\xi \in \mathbb{Z}[G]$ is in $I_{H}^{n}$, if and only if $¥(\xi)$ is in $\breve{I}_{H}^{n}$. In fact, if as in Lemma 2.10 we associate the isomorphisms $£_{n, G}$ and $£_{n, \breve{G}}$ respectively to the groups $G$ and $\breve{G}$, then

$$
¥ \circ £_{n, G}=\varpi^{n} £_{n, \breve{G}}
$$

and

$$
\mathrm{Val}_{\sigma, n, G / H} \circ ¥ \circ £_{n, G}=\varpi^{n} \mathrm{Val}_{\sigma, n, \breve{G} / H} \circ £_{n, \breve{G}} .
$$

\section{Stickelberger ELEMENTS AS REFinements OF THE EQUIVARIANT $L$-FUNCTIONS}

In this chapter, we review the definition of Stickelberger elements and show that they can be viewed as refinements of the equivariant $L$-functions.

\subsection{The Stickelberger elements.}

Definition 3.1 (Gro88, Tat84]). The Stickelberger element $\theta_{H^{\prime}}=\theta_{L / K^{\prime}}$ associated to the extension $L / K^{\prime}$ is the unique element of $\mathbb{Z}\left[H^{\prime}\right]$ such that for each continuous character $\psi$ of $H^{\prime}$,

$$
\psi\left(\theta_{H^{\prime}}\right)=L_{S\left(K^{\prime}\right), T\left(K^{\prime}\right)}(\psi, 0) .
$$

For the existence of the Stickelberger element, see [Gro88, Tat84].

In the case where $L / K$ is the constant $\mathbb{Z}_{p}$-extension, we can relate $\theta_{G}$ to $\Theta_{\Gamma, S, T}$ in the following way. First we note that the Galois group of the constant $\mathbb{Z}_{p}$-extension over $k$ can be identified with some $H^{\prime}$ such that $G$ can be identified with $\Gamma^{\prime} \times H^{\prime}$. This is actually the situation discussed in Section 2.4. We recall the notation used there, and, in particular, we have $\varpi=\left|H^{\prime} / H\right|$. Let $\sigma^{\prime}$ be the Frobenius of $H^{\prime}$. Then at every place $v \notin S$, the Frobenius element $[v] \in G$ can be expressed as the product $\gamma_{v}^{\prime} \cdot\left(\sigma^{\prime}\right)^{\operatorname{deg}(v)}, \gamma_{v}^{\prime} \in \Gamma^{\prime}$. For every natural number $d$, there are only finitely many $v$ with $\operatorname{deg}(v)=d$. From this we see that in the ring $\mathbb{Z}\left[\Gamma^{\prime}\right]\left[\left[\sigma^{\prime}\right]\right]$, the infinite product

$$
\Pi=\prod_{v \in T}(1-N(v) \cdot[v]) \prod_{v \notin S}(1-[v])^{-1}
$$

converges to a sum

$$
1+\sum_{d=1}^{\infty} a_{d}^{\prime} \cdot\left(\sigma^{\prime}\right)^{d}
$$

where each $a_{d}^{\prime}$ is an element in the group ring $\mathbb{Z}\left[\Gamma^{\prime}\right]$.

Lemma 3.2. Suppose $L / K$ is the constant $\mathbb{Z}_{p}$-extension and $\sigma$ is the Frobenius in $H=\operatorname{Gal}(L / K)$. Let the notation be as the above. Then the following holds:

(1) We have $a_{d}^{\prime}=0$, for almost all $d$, and also $\Pi=\theta_{G}$.

(2) If $¥\left(\theta_{G}\right)=\sum_{d} a_{d} \cdot(\sigma-1)^{d}, a_{d} \in \mathbb{Z}[\Gamma]$, then $\Theta_{\Gamma, S, T}=\sum_{d} a_{d} \cdot\left(q^{-s}-1\right)^{d}$. Furthermore, $a_{d}=0$, for $d=0, \ldots, m$, if and only if $\theta_{G} \in I_{H}^{m}$. In this case, we have

$$
\Theta_{\Gamma, S, T}(0)^{(m)}=a_{m}=\operatorname{Val}_{\sigma, m, \breve{G} / H}\left(¥\left(\left[\theta_{G}\right]_{(m, H)}\right)\right)=\varpi^{m} \cdot \operatorname{Val}_{\sigma, m, G / H}\left(\left[\theta_{G}\right]_{(m, H)}\right) .
$$


Proof. We first apply $¥$ to $\Pi$. Then we compare equations (11), (3) and (21) to see that part (1) and the first statement of part (2) hold. The rest is a consequence of Lemma 2.12.

3.2. Twisted Stickelberger elements. Suppose that $\left\{H_{\gamma} \mid \gamma \in \Gamma\right\}$ are the $H$ cosets of $G$. We view $\mathbb{Z}[G]$ as the ring of integer valued measures on $G$, and, for each $\gamma \in \Gamma$, let $\mathbb{Z}\left[H_{\gamma}\right]$ be the set of integer valued measures on the open subset $H_{\gamma}$. For a measure on $G$, we can restrict it to the open subset $H_{\gamma}$, and this defines the restriction map $\operatorname{res}_{H_{\gamma}}: \mathbb{Z}[G] \longrightarrow \mathbb{Z}\left[H_{\gamma}\right]$. Since $H_{\gamma}$ is also a closed subset of $G$, we can extend each measure in $\mathbb{Z}\left[H_{\gamma}\right]$ to a unique measure on $G$ vanishing outside $H_{\gamma}$. This extending of measures induces the injective map $\operatorname{ext}_{H_{\gamma}}: \mathbb{Z}\left[H_{\gamma}\right] \longrightarrow \mathbb{Z}[G]$.

Definition 3.3. Define, for each $\gamma \in \Gamma$ and each $\xi \in \mathbb{Z}[G]$, the $\gamma$-part of $\xi$ as $\xi_{\gamma}=\operatorname{ext}_{H_{\gamma}} \circ \operatorname{res}_{H_{\gamma}}(\xi)$. We have $\xi=\sum_{\gamma} \xi_{\gamma}$.

Definition 3.4. For $\chi \in \hat{\Gamma}$, the $\chi$-twist homomorphism is the ring homomorphism $[\chi]: \mathbb{Z}[\Gamma] \longrightarrow \mathcal{O}[\Gamma]$ which sends $\sum a_{\gamma} \gamma$ to $\sum a_{\gamma} \chi(\gamma) \gamma$. Also, the $\chi$-twist homomorphism from $\mathbb{Z}[G]$ to $\mathcal{O}[G]$ is the map sending $\xi=\sum_{\gamma \in \Gamma} \xi_{\gamma}$ to $\xi_{\chi}=\sum_{\gamma \in \Gamma} \chi(\gamma) \cdot \xi_{\gamma}$, and we also let $[\chi]$ denote this homomorphism.

Thus we have the twisted Stickelberger elements $\theta_{\chi}=[\chi]\left(\theta_{G}\right), \chi \in \hat{\Gamma}$. We have the following commutative diagram:

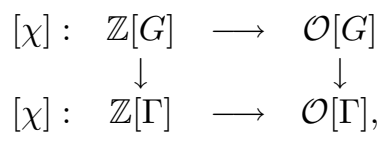

where both down-arrows are induced from the natural quotient map $G \longrightarrow \Gamma$.

\section{The ReFined REgUlator MAPS}

In this chapter we use the theory developed in Chapter 2 to define the refined regulator maps.

4.1. The global $\lambda$ map. Let $\mathbb{A}_{K^{\prime}}^{*}$ be the idèle group of $K^{\prime}$ and $\mathbb{A}_{K^{\prime}}^{*} \longrightarrow H^{\prime}$ be the norm residue map. Recall the map $\delta_{H^{\prime}}$ in (13). In a way similar to the one for constructing the map $\bar{\lambda}_{v, \Gamma}$ in (11), for each place $w$ of $K^{\prime}$ composite the natural embeddings $U\left(K^{\prime}\right) \longrightarrow K_{w}^{\prime *}$ and $K_{w}^{\prime *} \longrightarrow \mathbb{A}_{K^{\prime}}^{*}$ with $\delta_{H^{\prime}}$ to form

$$
\lambda_{w, H^{\prime}}: U\left(K^{\prime}\right) \longrightarrow K_{w}^{\prime *} \longrightarrow \mathbb{A}_{K^{\prime}}^{*} \longrightarrow H^{\prime} \stackrel{\delta_{H^{\prime}}}{\longrightarrow} I\left(H^{\prime}\right) / I\left(H^{\prime}\right)^{2} .
$$

Following [Gro90], we define the global $\lambda$ map.

Definition 4.1. Let

$$
\lambda_{H^{\prime}}: U\left(K^{\prime}\right) \longrightarrow X\left(K^{\prime}\right) \otimes I\left(H^{\prime}\right) / I\left(H^{\prime}\right)^{2} \subset Y\left(K^{\prime}\right) \otimes I\left(H^{\prime}\right) / I\left(H^{\prime}\right)^{2}
$$

be the homomorphism defined by

$$
\lambda_{H^{\prime}}(u)=\sum_{w \in S\left(K^{\prime}\right)} w \otimes \lambda_{w}(u), \text { for all } u \in U\left(K^{\prime}\right) .
$$

Note that we have, for $\gamma \in \Gamma^{\prime}$,

$$
\lambda_{\gamma w, H}(\gamma u)=\gamma \lambda_{w, H}(u),
$$

and therefore $\lambda_{H^{\prime}}$ is a $\Gamma^{\prime}$-equivariant homomorphism. Suppose that $H^{\prime} \subset H^{\prime \prime}=$ $\operatorname{Gal}\left(L / K^{\prime \prime}\right)$ are two subgroups of $G$ and $w^{\prime}, w^{\prime \prime}$ are respectively places of $K^{\prime}$ and $K^{\prime \prime}$ 
such that $w^{\prime \prime}=\left.w^{\prime}\right|_{K^{\prime \prime}}$. We identify $Y\left(K^{\prime \prime}\right)$ with a sub-module of $Y\left(K^{\prime}\right)$ by identifying $w^{\prime \prime}$ with the trace $\sum_{\sigma \in H^{\prime \prime} / H^{\prime}} \sigma w^{\prime} \in Y\left(K^{\prime}\right)$. Also, we identify $I\left(H^{\prime}\right) / I\left(H^{\prime}\right)^{2}$ with a subgroup of $I\left(H^{\prime \prime}\right) / I\left(H^{\prime \prime}\right)^{2}$, using the diagram

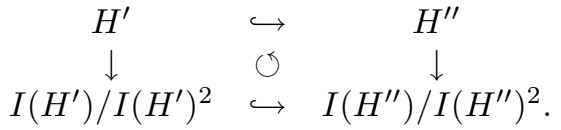

Then both $Y\left(K^{\prime}\right) \otimes I\left(H^{\prime}\right) / I\left(H^{\prime}\right)^{2}$ and $Y\left(K^{\prime \prime}\right) \otimes I\left(H^{\prime \prime}\right) / I\left(H^{\prime \prime}\right)^{2}$ can be viewed as sub-modules of $Y\left(K^{\prime}\right) \otimes I\left(H^{\prime \prime}\right) / I\left(H^{\prime \prime}\right)^{2}$ in which we have, for $u \in U\left(K^{\prime \prime}\right)$,

$$
\lambda_{H^{\prime \prime}}(u)=\lambda_{H^{\prime}}(u) .
$$

4.2. The refined regulator maps. Recall that for $\xi \in I(H)^{i}$ the symbol $[\xi]_{(i)}$ denotes its residue class modulo $I(H)^{i+1}$. Let $\Lambda^{n} U \longrightarrow \Lambda^{n}\left(X(K) \otimes I(H) / I(H)^{2}\right)$ be the homomorphism induced from $\lambda_{H}$ and let $\Lambda^{n}\left(X(K) \otimes I(H) / I(H)^{2}\right) \longrightarrow$ $\Lambda^{n} X(K) \otimes I(H)^{n} / I(H)^{n+1}$ be the $\Gamma$-equivariant homomorphism sending $\left(x_{1} \otimes\right.$ $\left.\left[h_{1}-1\right]_{(1)}\right) \wedge \cdots \wedge\left(x_{n} \otimes\left[h_{n}-1\right]_{(1)}\right)$ to $\left(x_{1} \wedge \cdots \wedge x_{n}\right) \otimes\left[\left(h_{1}-1\right) \cdots\left(h_{n}-1\right)\right]_{(n)}$. Then we define $\lambda_{H}^{(n)}$ as the composite

$$
\lambda_{H}^{(n)}: \Lambda^{n} U \longrightarrow \Lambda^{n}\left(X(K) \otimes I(H) / I(H)^{2}\right) \longrightarrow \Lambda^{n} X(K) \otimes I(H)^{n} / I(H)^{n+1} .
$$

Recall the notation in Chapter 1. In particular, the map $i^{(n)}$ induces a map

$$
i^{(n)} \otimes \operatorname{id}_{H}: \Lambda^{n} X(K) \otimes I(H)^{n} / I(H)^{n+1} \longrightarrow \Lambda^{n} Y(K) \otimes I(H)^{n} / I(H)^{n+1} .
$$

Also, for $\Psi \in \Lambda^{n} Y(K)^{*}$ the map $\iota(\Psi)$ induces a map

$$
\iota(\Psi) \otimes \operatorname{id}_{H}: \Lambda^{n} Y(K) \otimes I(H)^{n} / I(H)^{n+1} \longrightarrow \mathbb{Z}[\Gamma] \otimes I(H)^{n} / I(H)^{n+1} .
$$

We then make the composition $\left(\iota(\Psi) \otimes \operatorname{id}_{H}\right) \circ\left(i^{(n)} \otimes \operatorname{id}_{H}\right) \circ \lambda_{H}^{(n)}$ and extend it linearly to form the map $\mathcal{R}_{\Psi, H}^{\triangleright}: \mathbb{Q} \Lambda^{n} U \longrightarrow \mathbb{Q}[\Gamma] \otimes I(H)^{n} / I(H)^{n+1}$.

Definition 4.2. For an $\epsilon \in \mathbb{Q} \Lambda^{n} U$ such that $\mathcal{R}_{\Psi, \mathbb{H}}^{\triangleright}(\epsilon)$ is in $\mathbb{Z}[\Gamma] \otimes I(\mathbb{H})^{n} / I(\mathbb{H})^{n+1}$ and for the admissible Galois group $H$ with the natural quotient map $Q_{H}: \mathbb{H} \longrightarrow$ $H$, we define the refined regulator $\mathcal{R}_{\Psi, H}(\epsilon)$ as the image of $\mathcal{R}_{\Psi, \mathbb{H}}^{\triangleright}(\epsilon)$ under the map

$$
\mathbb{Z}[\Gamma] \otimes I(\mathbb{H})^{n} / I(\mathbb{H})^{n+1} \stackrel{\operatorname{id} \otimes Q_{H}}{\longrightarrow} \mathbb{Z}[\Gamma] \otimes I(H)^{n} / I(H)^{n+1} \stackrel{£_{n}}{\longrightarrow} I_{H}^{n} / I_{H}^{n+1} .
$$

Similar to the map $\iota$ defined in Chapter 1, we have the map

$$
\iota_{H}: \Lambda^{n} \operatorname{Hom}_{\Gamma}\left(U, \mathbb{Z}[\Gamma] \otimes I(H) / I(H)^{2}\right) \longrightarrow \operatorname{Hom}_{\Gamma}\left(\Lambda^{n} U, \mathbb{Z}[\Gamma] \otimes I(H)^{n} / I(H)^{n+1}\right),
$$

such that if $\phi_{1}, \ldots, \phi_{n} \in \operatorname{Hom}_{\Gamma}\left(U, \mathbb{Z}[\Gamma] \otimes I(H) / I(H)^{2}\right)$ and $u_{1}, \ldots, u_{n} \in U$, then

$$
\iota_{H}\left(\phi_{1} \wedge \cdots \wedge \phi_{n}\right)\left(u_{1} \wedge \cdots \wedge u_{n}\right)=\operatorname{det}\left(\phi_{i}\left(u_{j}\right)\right),
$$

where the determinant is computed by using the multiplication (28)

$$
\begin{aligned}
\mathbb{Z}[\Gamma] \otimes I(H)^{i} / I(H)^{i+1} \times \mathbb{Z}[\Gamma] \otimes I(H)^{j} / I(H)^{j+1} & \longrightarrow \mathbb{Z}[\Gamma] \otimes I(H)^{i+j} / I(H)^{i+j+1} \\
\left(a \otimes\left[\xi_{i}\right]_{(i)}, b \otimes\left[\xi_{j}\right]_{(j)}\right) & \mapsto a b \otimes\left[\xi_{i} \xi_{j}\right]_{(i+j)} .
\end{aligned}
$$

If $\psi_{i} \in Y(K)^{*}$, then $\mathcal{R}_{\psi_{i}, H}^{\triangleright} \in \operatorname{Hom}_{\Gamma}\left(U, \mathbb{Z}[\Gamma] \otimes I(H) / I(H)^{2}\right)$, and we have

$$
\mathcal{R}_{\psi_{1} \wedge \cdots \wedge \psi_{n}, H}^{\triangleright}=\iota_{H}\left(\mathcal{R}_{\psi_{1}, H}^{\triangleright} \wedge \cdots \wedge \mathcal{R}_{\psi_{n}, H}^{\triangleright}\right)
$$


Next, we will study the relation between $\mathcal{R}_{\Psi}$ and $\mathcal{R}_{\Psi, H}$. Our first goal is to show that $\mathcal{R}_{\Psi, H}(\epsilon)$ is defined for every $\epsilon \in \mathbb{Z}_{(p)} \Lambda_{0}^{n} U$.

Suppose $H \simeq \mathbb{Z}_{p}^{d}$ and $\left\{t_{1}, \ldots, t_{d}\right\}$ is a basis. Then $\mathbb{Z}[\Gamma] \otimes I(H) / I(H)^{2}$ can be identified with the direct sum $\mathbb{Z}_{p}[\Gamma] t_{1}+\cdots+\mathbb{Z}_{p}[\Gamma] t_{d}$. Let $\psi_{1}, \ldots, \psi_{n} \in Y(K)^{*}$. From the above construction, each $\mathcal{R}_{\psi_{i}, H}^{\triangleright}$ is a $\Gamma$-equivariant map from $\mathbb{Q} U$ to $\mathbb{Q}_{p}[\Gamma] t_{1}+$ $\cdots+\mathbb{Q}_{p}[\Gamma] t_{n}$ and we have $\mathcal{R}_{\psi_{i}, H}^{\triangleright}=\bigoplus_{j=1}^{d} \psi_{i j} t_{j}$, where each $\psi_{i j}$ is an element of $\mathbb{Z}_{(p)} U^{*}$. From the isomorphism (15) and Lemma 2.9, we see that $I(H)^{n} / I(H)^{n+1}$ is the $n$th symmetric tensor of $I(H) / I(H)^{2}$ and $\mathbb{Z}[\Gamma] \otimes I(H)^{n} / I(H)^{n+1}$ can be identified with the direct sum $\bigoplus_{n_{1}+\cdots+n_{d}=n} \mathbb{Z}_{p}[\Gamma] t_{1}^{n_{1}} \cdots \cdots t_{d}^{n_{d}}$. If $\Psi=\psi_{1} \wedge \cdots \wedge \psi_{n}$, then we have $\mathcal{R}_{\Psi, H}^{\triangleright}=\bigoplus_{n_{1}+\cdots+n_{d}=n} \Psi_{n_{1}, \ldots, n_{d}} t_{1}^{n_{1}} \cdots \cdots t_{d}^{n_{d}}$ where each $\Psi_{n_{1}, \ldots, n_{d}}$ is a $\Gamma$-equivariant map from $\mathbb{Q} \Lambda^{n} U$ to $\mathbb{Q}[\Gamma]$. In fact, if $\Xi_{n_{1}, \ldots, n_{d}}$ is the set consisting of all maps $\xi:\{1, \ldots, n\} \longrightarrow\{1, \ldots, d\}$ such that $\left|\xi^{-1}(i)\right|=n_{i}$, then from (29) we see that

$$
\Psi_{n_{1}, \ldots, n_{d}}=\sum_{\xi \in \Xi_{n_{1}, \ldots, n_{d}}} \iota\left(\psi_{1 \xi(1)} \wedge \cdots \wedge \psi_{n \xi(n)}\right) .
$$

For each $\epsilon \in \mathbb{Z}_{(p)} \Lambda_{0}^{n} U$, we have $\iota\left(\psi_{1 \xi(1)} \wedge \cdots \wedge \psi_{n \xi(n)}\right)(\epsilon) \in \mathbb{Z}_{p}[\Gamma]$, hence we must have $\mathcal{R}_{\Psi, H}^{\triangleright}(\epsilon) \in \mathbb{Z}[\Gamma] \otimes I(H)^{n} / I(H)^{n+1}$. Furthermore, since $\mathbb{H}$ is the projective limit of those $H$ which are finite free over $\mathbb{Z}_{p}$, we also have $\mathcal{R}_{\Psi, \mathbb{H}}^{\triangleright}(\epsilon) \in \mathbb{Z}[\Gamma] \otimes I(\mathbb{H})^{n} / I(\mathbb{H})^{n+1}$ for $\epsilon \in \mathbb{Z}_{(p)} \Lambda_{0}^{n} U$. The following lemma is proved.

Lemma 4.3. If $\Psi \in \Lambda^{n} Y(K)^{*}$, then the refined regulator $\mathcal{R}_{\Psi, H}(\epsilon)$ is defined for every $\epsilon \in \mathbb{Z}_{(p)} \Lambda_{0}^{n} U$.

Now we compare the refined regulator map with the old regulator map.

Lemma 4.4. Suppose $L / K$ is the constant $\mathbb{Z}_{p}$-extension and $\sigma$ is the Frobenius in $H=\operatorname{Gal}(L / K)$. Then for every $\Psi \in \Lambda^{n} Y(K)^{*}$ and every $\epsilon \in \Lambda_{0}^{n} U$

$$
\mathcal{R}_{\Psi}(\epsilon)=\operatorname{Val}_{\sigma, n}\left(¥\left(\mathcal{R}_{\Psi, H}(\epsilon)\right)\right) .
$$

Proof. It is easy to see that $\operatorname{Val}_{\sigma, 1}\left(¥\left(£_{1}\left(\lambda_{w, H}\right)\right)\right)=\lambda_{w}$, and from this we see that $\operatorname{Val}_{\sigma, 1}\left(¥\left(£_{1}\left(\mathcal{R}_{\psi_{i}, H}^{\triangleright}\left(u_{j}\right)\right)\right)\right)=\mathcal{R}_{\psi_{i}}\left(u_{j}\right)$. This proves the lemma for the $n=1$ case. The general case is proved by using (7) and (29).

4.3. The twisted regulators. Recall the $\chi$-twist homomorphisms defined in Definition 3.4.

Lemma 4.5. We have

$$
[\chi]\left(I_{H}^{n}\right) \subset I_{\mathcal{O}, H}^{n}
$$

Proof. This is due to (23).

By the abuse of notation, we also use $[\chi]$ to denote the induced homomorphism $I_{H}^{n} / I_{H}^{n+1} \longrightarrow I_{\mathcal{O}, H}^{n} / I_{\mathcal{O}, H}^{n+1}$.

Definition 4.6. For $\chi \in \hat{\Gamma}, \Psi \in \Lambda^{n} Y(K)^{*}$ and $\epsilon \in \mathbb{Z}_{(p)} \Lambda_{0}^{n} U$, define the $\chi$-twisted regulator

$$
\mathcal{R}_{\Psi, \chi, H}(\epsilon)=[\chi]\left(\mathcal{R}_{\Psi, H}(\epsilon)\right) \in I_{\mathcal{O}, H}^{n} / I_{\mathcal{O}, H}^{n+1}
$$


4.4. The universal property. In this section we study some special properties of the refined regulator map. We will show the injectivity as well as a universal property which says that every set of $n$ homomorphisms $\phi_{1}, \ldots, \phi_{n} \in \operatorname{Hom}_{\Gamma}\left(U, \mathbb{Z}_{p}[\Gamma]\right)$ can be obtained from the refined regulator map.

Let $w_{1}, \ldots, w_{n}, \eta$ and $w_{i}^{*}, \ldots, w_{n}^{*}$ be those defined in Chapter 1, Let $S^{\prime}$ be a finite set of places of $k$ such that $S \cap S^{\prime}=\emptyset$. For each $v \in S^{\prime}$ we arbitrarily choose a place $w$ of $K$ sitting over $v$. Let $S_{K}^{\prime}$ be the collection of these chosen places.

Lemma 4.7. For a place $w^{\prime} \in S_{K}^{\prime}$ let $\left[w^{\prime}\right] \in \mathbb{H}$ be the Frobenius element at $w^{\prime}$. If there are $u_{1}, \ldots, u_{n} \in U$ and $a_{w^{\prime}} \in \mathbb{Z}_{p}$ for each $w^{\prime} \in S_{K}^{\prime}$ such that in $I(\mathbb{H}) / I(\mathbb{H})^{2}$ the sum $\sum_{i=1}^{n} \lambda_{w_{i}, \mathbb{H}}\left(u_{i}\right)+\sum_{w^{\prime} \in S_{K}^{\prime}} a_{w^{\prime}} \delta_{\mathbb{H}}\left(\left[w^{\prime}\right]\right)$ is divisible by $p$, then in $U$ every $u_{i}$ is divisible by $p$.

Proof. We consider the maximal pro- $p$ abelian extension $\tilde{L} / K$ unramified outside $S(K)$. Denote $\tilde{\mathbb{G}}=\operatorname{Gal}(\tilde{L} / k)$ and $\tilde{\mathbb{H}}=\operatorname{Gal}(\tilde{L} / K)$. Then $\Gamma=\tilde{\mathbb{G}} / \tilde{\mathbb{H}}$ and $[\tilde{\mathbb{G}}, \tilde{\mathbb{G}}] \subset \tilde{\mathbb{H}}$. In particular, $\Gamma$ acts on $\tilde{\mathbb{H}}$ through conjugation. Let

$$
\mathbb{K}=[\tilde{\mathbb{G}}, \tilde{\mathbb{H}}]=\sum_{\gamma \in \Gamma}(1-\gamma) \tilde{\mathbb{H}},
$$

and denote $\mathbb{G}^{\prime}=\tilde{\mathbb{G}} / \mathbb{K}, \mathbb{H}^{\prime}=\tilde{\mathbb{H}} / \mathbb{K}$. Then, $\Gamma=\mathbb{G}^{\prime} / \mathbb{H}^{\prime}$ and $\mathbb{H}=\mathbb{H}^{\prime} /\left[\mathbb{G}^{\prime}, \mathbb{G}^{\prime}\right]$. As $\Gamma$ acts trivially on $\mathbb{H}^{\prime}$, we have

$$
\left[\mathbb{G}^{\prime}, \mathbb{H}^{\prime}\right]=\{\mathrm{id}\}
$$

Let $N=|\Gamma|$. Then for $f, g \in \mathbb{G}^{\prime}, f g^{N} f^{-1}=g^{N}$ and hence $f g f^{-1}=g h$ for some $h \in \mathbb{H}^{\prime}$ such that $h^{N}=i d$. By (31), the abelian group $\left[\mathbb{G}^{\prime}, \mathbb{G}^{\prime}\right]$ is generated by $N^{2}$ elements whose orders are divisors of $N$. Therefore, $\left[\mathbb{G}^{\prime}, \mathbb{G}^{\prime}\right]$ is finite and so is the index $C:=[[\tilde{\mathbb{G}}, \tilde{\mathbb{G}}]: \mathbb{K}]$. Let $p^{m}$ be the maximal $p$-power divisor of $C$.

Let $z_{i}=\left(z_{i, w}\right)_{w} \in \mathbb{A}_{K}^{*}$ be the idèle such that $z_{i, w}=u_{i}$ if $w=w_{i}$ and $z_{i, w}=1$ if $w \neq w_{i}$. If $\sum_{i=1}^{n} \lambda_{w_{i}, \mathbb{H}}\left(u_{i}\right)+\sum_{w^{\prime} \in S_{K}^{\prime}} a_{w^{\prime}} \delta_{\mathbb{H}}\left(\left[w^{\prime}\right]\right)$ is divisible by $p$ in $I(\mathbb{H}) / I(\mathbb{H})^{2}$, then by Class Field Theory, we can find $\alpha \in K^{*}, \beta \in \prod_{w \notin S(K)} \mathcal{O}_{w}^{*}, a \in \mathbb{A}_{K}^{*}$ and $b_{\gamma} \in \mathbb{A}_{K}^{*}$ for each $\gamma \in \Gamma$ such that

$$
\prod_{i=1}^{n} z_{i}^{C}=a^{p^{m+1}} \cdot \alpha \cdot \beta \cdot \prod_{w^{\prime} \in S_{K}^{\prime}} \pi_{w^{\prime}}^{C a_{w^{\prime}}} \cdot \prod_{\gamma \in \Gamma} \gamma b_{\gamma} / b_{\gamma} .
$$

Note that since $\left\{v_{1}, \ldots, v_{n}\right\}=S_{0} \varsubsetneqq S$, there is a place $v_{0} \in S \backslash S_{0}$. Taking the norm $N_{K / k}$, we see that at $v_{0}$ the norm $N_{K / k}(\alpha)$ is locally a $p^{m+1}$ th power. By Lemma 2.3. the element $N_{K / k}(\alpha)$ is a $p^{m+1}$ th power in $k^{*}$. This implies that each $N_{K / k}\left(z_{i}\right)$ is a $p$ th power idèle. Since $z_{i}$ is trivial away from $w_{i}$ and $v_{i}$ splits completely in $K, u_{i}=N_{K / k}\left(z_{i}\right)$ itself is a $p$ th power in $K_{w_{i}}^{*}$. Again, Lemma 2.3 implies that $u_{i}$ is a $p$ th power in $K^{*}$ and hence a $p$ th power in $U$.

Let $U_{i}=\mathbb{Z}_{p} \otimes U$, for $i=1, \ldots, n$. We extend each $\lambda_{w_{i}, \mathbb{H}}$ linearly to a map from $U_{i}$ to $I(\mathbb{H}) / I(\mathbb{H})^{2}$ and form the sum

$$
\lambda_{S_{0}, \mathbb{H}}:=\sum_{i=1}^{n} \lambda_{w_{i}, \mathbb{H}}: U_{1} \oplus \cdots \oplus U_{n} \longrightarrow I(\mathbb{H}) / I(\mathbb{H})^{2} .
$$

Let $\mathbb{U}$ be the image of $\lambda_{S_{0}, \mathbb{H}}$ and denote by $\mathbb{W}$ the $\mathbb{Z}_{p}$-sub-module of $I(H) / I(H)^{2}$ generated by the set $\left\{\delta_{\mathbb{H}}\left(\left[w^{\prime}\right]\right) \mid w^{\prime} \in S_{K}^{\prime}\right\}$. In view of Lemma 4.7, we have the following. 
Lemma 4.8. The map $\lambda_{S_{0}, \mathbb{H}}$ is injective and its image, denoted as $\mathbb{U}$, is a direct summand of $I(\mathbb{H}) / I(\mathbb{H})^{2}$ as a $\mathbb{Z}_{p}$-sub-module. Furthermore, we have $\mathbb{U} \cap \mathbb{W}=\{0\}$, and $(\mathbb{U}+\mathbb{W}) / \mathbb{W}$ is a direct summand of $\left(I(\mathbb{H}) / I(\mathbb{H})^{2}\right) / \mathbb{W}$.

As in Chapter 1, if $M$ is a $\mathbb{Z}[\Gamma]$-module, then there is a one-one correspondence $\phi \leftrightarrow \phi^{(i d)}$ between $\operatorname{Hom}_{\Gamma}\left(M, I_{H} / I_{H}^{2}\right)$ and $\operatorname{Hom}_{\mathbb{Z}}\left(M, I(H) / I(H)^{2}\right)$ such that

$$
\phi(m)=\sum_{\gamma \in \Gamma} \gamma \otimes \phi^{(i d)}\left(\gamma^{-1}(m)\right),
$$

for every $m \in M$. A similar correspondence holds if $M$ is a $\mathbb{Z}_{p}[\Gamma]$-module.

Recall that $\mathcal{R}_{w_{i}^{*}, \mathbb{H}}^{\triangleright}$ is formed by the linear extension of the composition $\left(w_{i}^{*} \otimes \mathrm{id}_{\mathbb{H}}\right) \circ\left(i \otimes \mathrm{id}_{\mathbb{H}}\right) \circ \lambda_{\mathbb{H}}$. Using the above notation, we easily find that

$$
\left(\left(w_{i}^{*} \otimes \mathrm{id}_{\mathbb{H}}\right) \circ\left(i \otimes \mathrm{id}_{\mathbb{H}}\right) \circ \lambda_{\mathbb{H}}\right)^{(\mathrm{id})}=\lambda_{w_{i}, \mathbb{H}} .
$$

Corollary 4.9. The maps $\mathcal{R}_{w_{1}^{*}, \mathbb{H}}^{\triangleright}, \ldots, \mathcal{R}_{w_{n}^{*}, \mathbb{H}}^{\triangleright} \in \operatorname{Hom}_{\Gamma}\left(\mathbb{Q} U, \mathbb{Q}[\Gamma] \otimes I(\mathbb{H}) / I(\mathbb{H})^{2}\right)$ are linearly independent over $\mathbb{Q}_{p}$.

Proof. Lemma 4.8 says $\left(\mathcal{R}_{w_{1}^{*}, \mathbb{H}}^{\triangleright}\right)^{(i d)}, \ldots,\left(\mathcal{R}_{w_{n}^{*}, \mathbb{H}}^{\triangleright}\right)^{(i d)}$ are linearly independent.

Corollary 4.10. Let the notation be as in Lemma 4.8. For every $\phi_{1}, \ldots, \phi_{n} \in$ $\operatorname{Hom}_{\Gamma}\left(U, \mathbb{Z}[\Gamma] \otimes I(H) / I(H)^{2}\right)$ there is a $\mathbb{Z}_{p}$-morphism $e: \mathbb{H} \longrightarrow H$ such that $\phi_{i}=$ $\delta_{H} \circ e \circ \delta_{\mathbb{H}}^{-1} \circ \mathcal{R}_{w_{i}^{*}, \mathbb{H}}^{\triangleright}$ for every $i$. Furthermore, e can be chosen such that $e(\mathbb{W})=0$.

Proof. Lemma 4.8 says that $e$ can be chosen to satisfy $\left(\phi_{i}\right)^{(i d)}=\delta_{H} \circ e \circ \delta_{\mathbb{H}}^{-1} \circ$ $\left(\mathcal{R}_{w_{i}^{*}, \mathbb{H}}^{\triangleright}\right)^{(i d)}$ for every $i$ and $e(\mathbb{W})=0$.

We make the identification $\mathbb{Z}_{p}=I\left(H_{0}\right) / I\left(H_{0}\right)^{2}=H_{0}:=\operatorname{Gal}\left(K \mathbb{F}_{q^{p \infty}} / K\right)$ and view $\mathbb{Z}$ as a subgroup of $I\left(H_{0}\right) / I\left(H_{0}\right)^{2}$. Then $U^{*}=\operatorname{Hom}_{\Gamma}(U, \mathbb{Z}[\Gamma])$ is identified as a subgroup of $\operatorname{Hom}_{\Gamma}\left(U, \mathbb{Z}[\Gamma] \otimes I\left(H_{0}\right) / I\left(H_{0}\right)^{2}\right)$.

Over $k$ choose a finite number of places not in $S$ such that with respect to the abelian extension $K / k$ the decomposition subgroups at these places generate the Galois group $\Gamma$. Let $S^{\prime}$ denote the set formed by these places. Suppose $\Phi=$ $\iota\left(\phi_{1} \wedge \cdots \wedge \phi_{n}\right) \neq 0$ with $\phi_{1}, \ldots, \phi_{n} \in U^{*} \subset \operatorname{Hom}_{\Gamma}\left(U, \mathbb{Z}[\Gamma] \otimes I\left(H_{0}\right) / I\left(H_{0}\right)^{2}\right)$. By Corollary 4.10, there is a $\mathbb{Z}_{p}$-morphism $e: \mathbb{H} \longrightarrow H_{0}$ such that

$$
\phi_{i}=\delta_{H_{0}} \circ e \circ \delta_{\mathbb{H}}^{-1} \circ \mathcal{R}_{w_{i}^{*}, \mathbb{H}}^{\triangleright}, \text { for } i=1, \ldots, n .
$$

Since $\Phi \neq 0$, the co-kernel of the morphism $e$ must be finite. Let $c$ be its order, and let $L_{1}$ be the fixed field of the kernel of $e$ and denote $H_{1}=\operatorname{Gal}\left(L_{1} / K\right)$, $G_{1}=\operatorname{Gal}\left(L_{1} / k\right)$. We have $e=j \circ Q$, where $Q: \mathbb{H} \longrightarrow H_{1}$ is the natural projection and $j: H_{1} \stackrel{\sim}{\longrightarrow} c H_{0} \hookrightarrow H_{0}$. Furthermore, Corollary 4.10 says that $e$ can be chosen such that every place of $K$ sitting over $S^{\prime}$ splits completely over $L_{1} / K$. Thus, over $L_{1} / k$ the decomposition subgroup at each place in $S^{\prime}$ is a finite subgroup of $G_{1}$, and these decomposition groups generate a finite group which, under the natural projection $G_{1} \longrightarrow G_{1} / H_{1}=\Gamma$, is isomorphic to $\Gamma$. This means that $G_{1}$ is the direct product $\Gamma \times H_{1}$.

Let $\sigma \in H_{1}$ be the generator such that $j(\sigma)$ is $c$ times the Frobenius in $H_{0}$, and let $t=\sigma-1 \in \mathbb{Z}\left[H_{1}\right]$. Then we have

$$
F_{p}\left[G_{1}\right]=F_{p}[\Gamma][[t]]
$$


and by Lemma 2.10 the $n$th relative augmentation ideal $I_{F_{p}, H_{1}}^{n}$ is just the principal ideal $\left(t^{n}\right)$. If $\epsilon_{1}$ is an element in $\mathbb{Q} \Lambda^{n} U$, then $£_{n}\left(\mathcal{R}_{\eta, H_{1}}^{\triangleright}\left(\epsilon_{1}\right)\right)=a_{n} t^{n}$ for some $a_{n} \in F_{p}[\Gamma]$ and we have

$$
\Phi\left(\epsilon_{1}\right)=\iota\left(\phi_{1} \wedge \cdots \wedge \phi_{n}\right)\left(\epsilon_{1}\right)=a_{n}=\operatorname{Val}_{\sigma, n, G / H_{1}}\left(£_{n}\left(\mathcal{R}_{\eta, H_{1}}^{\triangleright}\left(\epsilon_{1}\right)\right)\right) .
$$

In particular, if $\mathcal{R}_{\eta, \mathbb{H}}\left(\epsilon_{1}\right)$ is defined, then $\Phi\left(\epsilon_{1}\right) \in \mathbb{Z}_{p}[\Gamma]$, and hence $\epsilon_{1}$ is an element of $\mathbb{Z}_{(p)} \Lambda_{0}^{n} U$. Thus we have proved the following corollary.

Corollary 4.11. If $\mathcal{R}_{\eta, \mathbb{H}}(\epsilon)$ is defined, then $\epsilon$ is indeed an element of $\mathbb{Z}_{(p)} \Lambda_{0}^{n} U$. If the notation is as above, then we have

$$
\Phi(\epsilon)=\operatorname{Val}_{\sigma, n, G / H_{1}}\left(\mathcal{R}_{\eta, H_{1}}(\epsilon)\right) .
$$

Lemma 4.12. Suppose that $n$ and $\eta$ are as in Conjecture 1.4. Then the following are true:

(1) The map $\mathcal{R}_{\eta, \mathbb{H}}^{\triangleright}: \mathbb{Q} \Lambda^{n} U \longrightarrow \mathbb{Q} I_{\mathbb{H}}^{n} / I_{\mathbb{H}}^{n+1}$ is injective.

(2) For each $\chi \in \hat{\Gamma}$, the map $\mathcal{R}_{\eta, \chi, \mathbb{H}}^{\triangleright}=:[\chi] \circ \mathcal{R}_{\eta, \mathbb{H}}^{\triangleright}: \mathbb{Q} \Lambda^{n} U \longrightarrow F I_{\mathbb{H}}^{n} / I_{\mathbb{H}}^{n+1}$ is injective.

Proof. The $\chi$-twist map $[\chi]: \mathbb{Q} I_{\mathbb{H}}^{n} / I_{\mathbb{H}}^{n+1} \longrightarrow F I_{\mathbb{H}}^{n} / I_{\mathbb{H}}^{n+1}$ is injective, and hence part (2) is a consequence of part (1).

To prove part (1), we first apply Corollary 4.9 and choose an admissible $H_{0}$ such that $H_{0} \simeq \mathbb{Z}_{p}^{d}$ for some $d$ and the subspaces $\mathcal{R}_{w_{1}^{*}, H_{0}}^{\triangleright}(U), \ldots, \mathcal{R}_{w_{n}^{*}, H_{0}}^{\triangleright}(U) \subset$ $\mathbb{Q}[\Gamma] \otimes I\left(H_{0}\right) / I\left(H_{0}\right)^{2}$ are linearly independent over $\mathbb{Q}_{p}$. To simplify the notation, put $W=\overline{\mathbb{Q}}_{p} \otimes U, V=\overline{\mathbb{Q}}_{p} \otimes_{\mathbb{Z}_{p}} I_{H_{0}} / I_{H_{0}}^{2}$, and linearly extend each $\mathcal{R}_{w_{i}^{*}, H_{0}}^{\triangleright}$ to the map $\mathcal{R}_{i}: W \longrightarrow V$.

From equation (29), we see that it is enough to show that the map

$$
\iota_{H_{0}}\left(\mathcal{R}_{1} \wedge \cdots \wedge \mathcal{R}_{n}\right): \Lambda^{n} W \longrightarrow \overline{\mathbb{Q}}_{p} \otimes_{\mathbb{Z}_{p}} I_{H_{0}}^{n} / I_{H_{0}}^{n+1}
$$

is injective.

By Lemma 2.10 and equation (14), in the category of $\overline{\mathbb{Q}}_{p}[\Gamma]$-modules, $\overline{\mathbb{Q}}_{p} \otimes_{\mathbb{Z}_{p}}$ $I_{H_{0}}^{n} / I_{H_{0}}^{n+1}$ is nothing but the $n$th symmetric tensor of $V$. Without loss of generality, we can assume that $V=\mathcal{R}_{1}(W) \oplus \cdots \oplus \mathcal{R}_{n}(W)$. If

$$
W=W_{1}+\cdots+W_{m}
$$

is the decomposition of $W$ into irreducible $\overline{\mathbb{Q}}_{p}[\Gamma]$-modules, then $\Lambda^{n} W$ is decomposed into the direct sum $\bigoplus_{A} W_{A}$, where, associated to each $A=\left\{i_{1}, \ldots, i_{n}\right\} \subset\{1, \ldots, m\}$ such that $|A|=n, W_{A}$ is the exterior tensor of $W_{i_{1}}, \ldots, W_{i_{n}}$. Also, $\overline{\mathbb{Q}}_{p} \otimes_{\mathbb{Z}_{p}} I_{H_{0}}^{n} / I_{H_{0}}^{n+1}$ is decomposed into the direct sum $\bigoplus_{A, \sigma} V_{A, \sigma}$, where associated to each pair $(A, \sigma)$ with $A$ as above and $\sigma \in S_{n}$, the symmetric group of $n$ elements, $V_{A, \sigma}$ is the symmetric tensor of $\mathcal{R}_{1}\left(W_{\sigma\left(i_{1}\right)}\right), \ldots, \mathcal{R}_{i_{n}}\left(W_{\sigma\left(i_{n}\right)}\right)$. By (27), the homomorphism $\iota_{H_{0}}\left(\mathcal{R}_{1} \wedge \cdots \wedge \mathcal{R}_{n}\right)$ is injective on each $W_{A}$, and it sends $W_{A}$ into $\sum_{\sigma \in S_{n}} V_{A, \sigma}$. Therefore, it is injective on $\Lambda^{n} W$.

\section{THE MAIN THEOREM}

In this chapter, we state our main theorem and show that it implies Theorem 1.5 and Theorem 1.7. We also state a twisted version of it. 


\subsection{The main theorem.}

Theorem 5.1. Let the notation be as in Corollary 1.4, Then for every admissible $H$, the Stickelberger element $\theta_{G} \in I_{H}^{n}$. Furthermore, there is a unique $\epsilon \in \mathbb{Z}_{(p)} \Lambda_{S, T}^{n}$ such that for every admissible $H$

$$
\mathcal{R}_{\eta, H}(\epsilon)=\left[\theta_{G}\right]_{(n, H)} .
$$

Note that the uniqueness of $\epsilon$ follows from (36) and Lemma 4.12

Now we show that this main theorem implies Theorem 1.5.

Proof of Theorem 1.5. Let $H=\operatorname{Gal}\left(K \mathbb{F}_{q^{p}} / K\right)$ and let $\sigma$ be the Frobenius, and apply $\mathrm{Val}_{\sigma, n, G / H} \circ ¥$ to both sides of (36). Then we use Lemma 3.2 and Lemma 4.4 .

5.2. The conjecture of Gross. To complete the proof of Theorem 1.7 we need to use some results concerning a conjecture of Gross, which will be discussed in this section. This conjecture can be viewed as a refinement of the class number formula in which $\operatorname{det}_{H^{\prime}}$, a refined regulator of Gross, is involved.

In Gro88, this refined regulator is defined as an element in $I^{n} / I^{n+1}$. We instead choose to adopt Tate's definition [Tat97] and define the refined regulator as an element in the group ring. Here we describe Tate's definition of the refined regulator.

For a place $w \in S\left(K^{\prime}\right)$ let $\lambda_{w, H^{\prime}}: U\left(K^{\prime}\right) \longrightarrow I\left(H^{\prime}\right) / I\left(H^{\prime}\right)^{2}$ be the map in (24), and let $\delta_{H^{\prime}}$ be the map in (13). Suppose $w_{1}^{\prime}, \ldots, w_{r_{K^{\prime}}}^{\prime}$ are distinct places in $S\left(K^{\prime}\right)$ and $u_{1}, \ldots, u_{r_{K^{\prime}}}$ is a $\mathbb{Z}$-basis of $U\left(K^{\prime}\right)$, and assume that the ordering of them are chosen such that the classical regulator formed by them is positive. Then the refined regulator of Gross is defined as

$$
\operatorname{det}_{H^{\prime}}=\operatorname{det}_{1 \leq i, j \leq r_{K^{\prime}}}\left(\delta_{H^{\prime}}^{-1}\left(\lambda_{w_{i}^{\prime}, H^{\prime}}\left(u_{j}\right)\right)-1\right) \in I\left(H^{\prime}\right)^{r_{K^{\prime}}} .
$$

For each $K^{\prime}$, recall that $h_{K^{\prime}, S\left(K^{\prime}\right), T\left(K^{\prime}\right)}$ is the modified class number defined in (44).

Conjecture 5.2 (Gross). We have $\theta_{H^{\prime}} \in I\left(H^{\prime}\right)^{r_{K^{\prime}}}$ and

$$
\theta_{H^{\prime}} \equiv h_{K^{\prime}, S\left(K^{\prime}\right), T\left(K^{\prime}\right)} \cdot \operatorname{det}_{H^{\prime}} \quad\left(\bmod I\left(H^{\prime}\right)^{r_{K^{\prime}}+1}\right) .
$$

Evidences and related discussions of this conjecture can be found in many documents. For examples, see Aok91, Aok03, Bun02, Bun04, Bun07, BnL04, Dar95, Hay88, Hua04, Lee97, Lee02, Lee04, Rei02, Tat97, Tat04, Tan95, Tan04, Yam89. We will need the following result from Tan95.

Theorem 5.3. We have $\theta_{H^{\prime}} \in I_{p}\left(H^{\prime}\right)^{r_{K^{\prime}}}$ and

$$
\theta_{H^{\prime}} \equiv h_{K^{\prime}, S\left(K^{\prime}\right), T\left(K^{\prime}\right)} \cdot \operatorname{det}_{H^{\prime}} \quad\left(\bmod I_{p}\left(H^{\prime}\right)^{r_{K^{\prime}}+1}\right) .
$$

Note that in [Tan95, this theorem is proved only for the case where $H^{\prime}$ is a pro- $p$ group. But, since $\theta_{H^{\prime}}$ is known to be in $I\left(H^{\prime}\right)$ and if $H^{\prime}=H_{p}^{\prime} \times H_{0}^{\prime}$ is the decomposition into the direct product of the pro- $p$ part, $H_{p}^{\prime}$, and the non- $p$ part, $H_{0}^{\prime}$, then for each $m \geq 1$,

$$
I_{p}\left(H^{\prime}\right)^{m} / I_{p}\left(H^{\prime}\right)^{m+1}=I_{p}\left(H_{p}^{\prime}\right)^{m} / I_{p}\left(H_{p}^{\prime}\right)^{m+1} \times I_{p}\left(H_{0}^{\prime}\right)^{m} / I_{p}\left(H_{0}^{\prime}\right)^{m+1},
$$

with $I_{p}\left(H_{0}^{\prime}\right)^{m} / I_{p}\left(H_{0}^{\prime}\right)^{m+1}=0$. Therefore, the theorem holds for general $H^{\prime}$.

We are ready to show that Theorem 5.1 implies Theorem 1.7 
Proof of Theorem 1.7. We recall the notation in the proof of Corollary 4.11. Thus the admissible Galois groups $H_{0}$ and $H_{1}$ are chosen and we have $G_{1}=\Gamma \times H_{1}$, also $\sigma \in H_{1}$ is a generator, and with $t=\sigma-1 \in \mathbb{Z}\left[H_{1}\right]$ we have

$$
\mathbb{Z}_{p}\left[G_{1}\right]=\mathbb{Z}_{p}[\Gamma][[t]]
$$

such that the $n$th relative augmentation ideal $I_{p, H_{1}}^{n}$ is just the principal ideal $\left(t^{n}\right)$. The first part of Theorem 5.1 says that $\theta_{G_{1}} \in I_{p, H_{1}}^{n}$ and hence

$$
\theta_{G_{1}}=a_{n} t^{n}+\cdots+a_{i} t^{i}+\ldots, \quad a_{i} \in \mathbb{Z}_{p}[\Gamma],
$$

from which we get $a_{n}=\operatorname{Val}_{\sigma, n, G / H_{1}}\left(\left[\theta_{G_{1}}\right]_{\left(n, H_{1}\right)}\right)$. Now Corollary 4.11 and the second part of Theorem 5.1 say that

$$
\Phi(\epsilon)=\operatorname{Val}_{\sigma, n, G / H_{1}}\left(\mathcal{R}_{\eta, H_{1}}(\epsilon)\right)=\operatorname{Val}_{\sigma, n, G / H_{1}}\left(\left[\theta_{G_{1}}\right]_{\left(n, H_{1}\right)}\right)=a_{n} .
$$

If $v_{i} \in S_{0}$, then it splits completely over $K$ and hence for $u \in U(k)$ the image $\lambda_{v_{i}, G_{1}}(u) \in I\left(H_{1}\right) / I\left(H_{1}\right)^{2} \subset I\left(G_{1}\right) / I\left(G_{1}\right)^{2}$. This implies that $\operatorname{det}_{G_{1}}$ is in $I\left(G_{1}\right)^{r-n} I\left(H_{1}\right)^{n}$ and hence

$$
\operatorname{det}_{G_{1}}=b_{n} t^{n}+\cdots+b_{i} t^{i}+\cdots .
$$

Since $G_{1}$ is the direct product of $\Gamma$ and $H_{1}$, the augmentation ideal $I_{p}\left(G_{1}\right)$ is generated by $t$ and $I_{p}(\Gamma)$. Therefore, an element

$$
\xi=c_{0}+c_{1} t+\cdots+c_{i} t^{i}+\cdots \in \mathbb{Z}_{p}\left[G_{1}\right]
$$

is in $I_{p}\left(G_{1}\right)^{m}$ if and only if $c_{i} \in I_{p}(\Gamma)^{m-i}$ for every $i \leq m$. This together with (39), (41) and Theorem 5.3 implies that for $i=n, \ldots, r_{k}$

$$
a_{i} \equiv b_{i} \quad\left(\bmod I_{p}(\Gamma)^{r_{k}-i+1}\right) .
$$

From (33), we see that for $i=1, \ldots, n$ and $u \in U(k)$

$$
\delta_{G_{1}}^{-1}\left(\lambda_{v_{i}, G_{1}}(u)\right)-1=\phi_{i}^{(i d)}(u) t .
$$

For $i=n+1, \ldots, r_{k}$ and $u \in U(k)$, we have

$$
\delta_{G_{1}}^{-1}\left(\lambda_{v_{i}, G_{1}}(u)\right)=\bar{\lambda}_{v_{i}, \Gamma}(u) \cdot \sigma^{\alpha}, \text { for some } \alpha \in \mathbb{Z}_{p},
$$

and hence

$$
\delta_{G_{1}}^{-1}\left(\lambda_{v_{i}, G_{1}}(u)\right)-1 \equiv \bar{\lambda}_{v_{i}, \Gamma}(u)-1+\alpha t \quad\left(\bmod I_{p}\left(G_{1}\right) I_{p}\left(H_{1}\right)\right) .
$$

Therefore,

$$
\operatorname{det}_{G_{1}}=\operatorname{Reg}_{\Gamma}^{\Phi} t^{n}+b_{n+1} t^{n+1}+\cdots
$$

This together with (40) and (42) implies the theorem.

\subsection{A twisted version.}

Theorem 5.4. Let the notation be as in Corollary 1.4. Then for every admissible $H$ and every $\chi \in \hat{\Gamma}$, the twisted Stickelberger element $\theta_{\chi} \in I_{\mathcal{O}, H}^{n}$. Furthermore, there is a unique $\epsilon \in \mathbb{Z}_{(p)} \Lambda_{S, T}^{n}$ such that for every admissible $H$ and every $\chi \in \hat{\Gamma}$,

$$
\mathcal{R}_{\eta, \chi, H}=\left[\theta_{\chi}\right]_{(n, H)} .
$$

Proof. We only need to use Theorem 5.1 and then apply the $\chi$-twisted map to the Stickelberger element and the refined regulator. The uniqueness is a consequence of Lemma 4.12. 


\section{The DeCOMposition OF THE REFined REgulator}

6.1. The canonical pairing. In this chapter, we show that $\operatorname{det}_{\mathbb{H}}$, the regulator of Gross, is decomposed into a product of irreducible factors. To do so, it is helpful to consider the dual version of the homomorphism $\lambda_{H^{\prime}}$, which can be described as a pairing.

Definition 6.1. Let

$$
\begin{aligned}
Y^{\prime}\left(K^{\prime}\right) & =\operatorname{Hom}_{\mathbb{Z}}\left(Y\left(K^{\prime}\right), \mathbb{Z}\right), \\
X^{\prime}\left(K^{\prime}\right) & =\operatorname{Hom}_{\mathbb{Z}}\left(X\left(K^{\prime}\right), \mathbb{Z}\right),
\end{aligned}
$$

and let

$$
\langle\cdot, \cdot\rangle_{H^{\prime}}: U\left(K^{\prime}\right) \times Y^{\prime}\left(K^{\prime}\right) \longrightarrow I\left(H^{\prime}\right) / I\left(H^{\prime}\right)^{2}
$$

be the pairing defined by

$$
\langle u, \phi\rangle_{H^{\prime}}=\sum_{w \in S\left(K^{\prime}\right)} \phi(w) \cdot \lambda_{w}(u), \text { for all } u \in U\left(K^{\prime}\right), \phi \in Y^{\prime}\left(K^{\prime}\right) .
$$

This pairing factors through a unique pairing on $U\left(K^{\prime}\right) \times X^{\prime}\left(K^{\prime}\right)$, which, by the abuse of notation, will also be denoted as $\langle\cdot, \cdot\rangle_{H^{\prime}}$.

Following from (26), for $H^{\prime} \subset H^{\prime \prime}$, we have

$$
\left\langle u,\left.\phi\right|_{Y^{\prime}\left(K^{\prime \prime}\right)}\right\rangle_{H^{\prime \prime}}=\langle u, \phi\rangle_{H^{\prime}} \text {, for all } u \in U\left(K^{\prime \prime}\right), \phi \in Y^{\prime}\left(K^{\prime}\right) \text {. }
$$

Directly from the definition, we have

$$
\left\langle{ }^{\sigma} u,{ }^{\sigma} \phi\right\rangle_{H^{\prime}}=\langle u, \phi\rangle_{H^{\prime}} \text {, for all } \sigma \in G, u \in U\left(K^{\prime}\right), \phi \in Y^{\prime}\left(K^{\prime}\right),
$$

where

$$
{ }^{\sigma} \phi(w)=\phi\left(\sigma^{-1}(w)\right) .
$$

If $A=\left\{a_{i}\right\}_{i=1, \ldots, r_{K^{\prime}}}, B=\left\{b_{j}\right\}_{j=1, \ldots, r_{K^{\prime}}}$ are $\mathbb{Z}$-bases of $U\left(K^{\prime}\right)$ and $X^{\prime}\left(K^{\prime}\right)$, then as usual the associated discriminant of the pairing $\langle\cdot, \cdot\rangle_{H^{\prime}}$ is defined as

$$
=\quad \begin{array}{ll}
\operatorname{det}\left(\left\langle a_{i}, b_{j}\right\rangle\right)_{i, j=1, \ldots, r_{K^{\prime}}} \\
\sum_{\pi \in S_{r^{\prime}}} \operatorname{sign}(\pi)\left\langle a_{1}, b_{\pi(1)}\right\rangle \cdots\left\langle a_{r_{K^{\prime}}}, b_{\pi\left(r_{K^{\prime}}\right)}\right\rangle,
\end{array}
$$

which is considered as an element of $I\left(H^{\prime}\right)^{r_{K^{\prime}}} / I\left(H^{\prime}\right)^{r_{K^{\prime}}+1}$. This discriminant is independent of the choice of the bases up to \pm 1 . The following lemma is obvious.

Lemma 6.2. Let $w_{1}^{\prime}, \ldots, w_{r_{K^{\prime}}}^{\prime} \in S\left(K^{\prime}\right)$ and the basis $u_{1}, \ldots, u_{r_{K^{\prime}}} \in U\left(K^{\prime}\right)$ be as in the definition of $\operatorname{det}_{H^{\prime}}$. Choose the basis $\phi_{1}, \ldots, \phi_{r_{K^{\prime}}} \in X^{\prime}\left(K^{\prime}\right)$ such that

$$
\phi_{i}\left(a_{1} w_{1}^{\prime}+\cdots+a_{i} w_{i}^{\prime}+\cdots+a_{r_{K^{\prime}}} w_{r_{K^{\prime}}}^{\prime}\right)=a_{i} .
$$

Then the associated discriminant of $\langle\cdot, \cdot\rangle_{H^{\prime}}$ equals $\left[\operatorname{det}_{H^{\prime}}\right]_{(n)}$.

If $L / K$ is the constant $\mathbb{Z}_{p}$-extension and $\sigma \in H$ is the Frobenius, then $\operatorname{Val}_{\sigma, r}\left(\left[\operatorname{det}_{H}\right]_{(r)}\right)$ is just the classical regulator of $U$. Therefore, the following lemma holds.

Lemma 6.3. Suppose that $L / K$ is the constant $\mathbb{Z}_{p}$-extension. Then the pairing

$$
U \times X^{\prime}(K): \stackrel{\langle\cdot \cdot\rangle_{H}}{\longrightarrow} I(H) / I(H)^{2} \stackrel{\delta_{H}^{-1}}{\longrightarrow} H=\mathbb{Z}_{p}
$$

has all its values in $\mathbb{Z} \subset H$. Furthermore, this pairing induces a perfect pairing on $\mathbb{Q} U \times \mathbb{Q} X^{\prime}(K)$. 
The $\chi$-eigenspace of $F_{p} X^{\prime}(K)$ will be denoted as $X_{\chi}^{\prime}$. We have

$$
F_{p} X^{\prime}(K)=\bigoplus_{\chi \in \hat{\Gamma}} X_{\chi}^{\prime} .
$$

We denote by $\langle\cdot, \cdot\rangle_{H, p}$ the induced pairing on $F_{p} U \times F_{p} X^{\prime}(K)$. Note that by equation (46) and Lemma [6.3, we have a duality between the $F_{p}[\Gamma]$-modules $U_{\chi}$ and $X_{\chi^{-1}}^{\prime}$, where $U_{\chi}$ is the $\chi$-eigenspace of $F_{p} \otimes U$ with $\operatorname{dim}_{F_{p}} U_{\chi}=r_{\chi}$.

Definition 6.4. Let $\langle\cdot, \cdot\rangle_{\chi}$ denote the restriction of $\langle\cdot, \cdot\rangle_{H, p}$ to $U_{\chi} \times X_{\chi^{-1}}^{\prime}$. If $A_{\chi}=\left\{c_{1}, \ldots, c_{r_{\chi}}\right\}$ and $B_{\chi^{-1}}=\left\{d_{1}, \ldots, d_{r_{\chi}}\right\}$ are $F_{p^{-}}$bases of $U_{\chi}$ and $X_{\chi^{-1}}^{\prime}$, then define

$$
\begin{aligned}
{\left[\operatorname{det}_{\chi}\right]_{\left(r_{\chi}\right)} } & =\operatorname{det}\left(\left\langle c_{i}, d_{j}\right\rangle\right)_{i, j=1, \ldots, r_{\chi}} \\
& =\sum_{\pi \in S_{r_{\chi}}} \operatorname{sign}(\pi)\left\langle c_{1}, d_{\pi(1)}\right\rangle \cdots\left\langle c_{r_{\chi}}, d_{\pi\left(r_{\chi}\right)}\right\rangle,
\end{aligned}
$$

which is viewed as an element in $I_{F_{p}}(H)^{r_{\chi}} / I_{F_{p}}(H)^{r_{\chi}+1}$.

This discriminant is independent of the choice of bases up to elements of $F_{p}^{*}$.

6.2. $(K / k, S)$-extensions. The main result of this chapter is Proposition 6.15, which concerns the decomposition of the refined regulator $\operatorname{det}_{H^{\prime}}$. For this purpose, we need to consider Galois extensions that might not be admissible.

Definition 6.5. Let $\tilde{L} / k$ be a Galois extension. Then $\tilde{L} / k$ is called a $(K / k, S)$ extension if $K \subset \tilde{L}$ and the associated field extension $\tilde{L} / K$ is pro- $p$, abelian and unramified outside $S(K)$. If $\tilde{L}$ contains the given field $L$, then we say that $\tilde{L} / k$ is a $(K / k, S)$-extension of $L / k$ and $\tilde{H}:=\operatorname{Gal}(\tilde{L} / K)$ is a $(K / k, S)$-extension of $H=\operatorname{Gal}(L / K)$. We say that $\tilde{L} / k$ is a strict $(K / k, S)$-extension of $L / k$ and $\tilde{H}$ is a strict $(K / k, S)$-extension of $H$ if $L / k$ is the maximal admissible field extension contained in $\tilde{L} / k$.

If $\tilde{H}$ is a strict $(K / k, S)$-extension of $H$, then the natural quotient map $\tilde{H} \longrightarrow H$ factors through

$$
\tilde{H} \longrightarrow \tilde{H} / \sum_{\gamma \in \Gamma}(1-\gamma) \tilde{H} \longrightarrow H,
$$

where the second arrow has a finite kernel.

Lemma 6.6. Suppose that $L^{\prime} / K$ is a pro-p abelian extension unramified outside $S(K)$. Then there exists an extension $\tilde{L} / k$, which contains $L^{\prime} / k$ and is a $(K / k, S)$ extension of $L / k$. If both $H$ and $\operatorname{Gal}\left(L^{\prime} / K\right)$ are finitely generated over $\mathbb{Z}_{p}$, then $\tilde{L} / k$ can be chosen such that $\operatorname{Gal}(\tilde{L} / K)$ is finite free over $\mathbb{Z}_{p}$ and its maximal admissible quotient is also finite free over $\mathbb{Z}_{p}$.

Proof. For the first statement, we let $\tilde{L}$ be the compositum of $L$ with all the fields ${ }^{\sigma} L^{\prime}, \sigma \in \operatorname{Gal}\left(\bar{k}^{s e p} / k\right)$.

If the conditions of the second statement hold, then $\operatorname{Gal}(\tilde{L} / K)$ is finitely generated over $\mathbb{Z}_{p}$. Lemma 2.4 (for $\mathcal{K}=K$ and $\mathcal{S}=S(K)$ ) then implies that there is an abelian extension $M / K$ which is unramified outside $S(K)$, with the Galois group finite free over $\mathbb{Z}_{p}$, and contains $\tilde{L} / K$. Let $M^{\prime}$ be the compositum of all the fields ${ }^{\sigma} M, \sigma \in \operatorname{Gal}\left(\bar{k}^{\text {sep }} / k\right)$. Then $M^{\prime} / k$ is a $(K / k, S)$-extension and $\operatorname{Gal}\left(M^{\prime} / K\right)$ is finite free over $\mathbb{Z}_{p}$. The maximal admissible quotient of $\operatorname{Gal}\left(M^{\prime} / K\right)$ might not be free 
over $\mathbb{Z}_{p}$. By Lemma 2.2, we can chose an admissible extension $L^{\prime \prime} / k$ such that the Galois group $\operatorname{Gal}\left(L^{\prime \prime} / K\right)$ is finite free over $\mathbb{Z}_{p}$ and contains the maximal admissible quotient of $\operatorname{Gal}\left(M^{\prime} / K\right)$. We complete the proof by replacing $\tilde{L}$ by $M^{\prime} L^{\prime \prime}$.

\subsection{Universal pairings.}

Definition 6.7. Let $A, B$ and $C$ be $F_{p}$-vector spaces and let $\left\{a_{i}\right\}_{i},\left\{b_{j}\right\}_{j}$ be bases of $A$ and $B$. A pairing of $F_{p}$-spaces

$$
\Phi: A \times B \longrightarrow C
$$

is said to be universal if the set $\left\{\Phi\left(a_{i}, b_{j}\right)\right\}_{i, j}$ is linearly independent over $F_{p}$.

In other words, $\Phi$ is universal if and only if its image generates a subspace which is canonically isomorphic to $A \otimes_{F_{p}} B$.

Suppose that $\tilde{L} / k$ is a $(K / k, S)$-extension and $\tilde{H}=\operatorname{Gal}(\tilde{L} / K)$. Then $\tilde{H}$ is abelian. We can use (44) and define the pairing

$$
\langle\cdot, \cdot\rangle_{\tilde{H}}: U \times X^{\prime}(K) \longrightarrow I(\tilde{H}) / I(\tilde{H})^{2} .
$$

Then the pairing $\langle\cdot, \cdot\rangle_{\tilde{H}}$ is $\Gamma$-equivariant in the sense that

$$
\left\langle{ }^{\gamma} u,{ }^{\gamma} \phi\right\rangle_{\tilde{H}}={ }^{\gamma}\langle u, \phi\rangle_{\tilde{H}} \text {, for all } u \in U, \phi \in X^{\prime}(K), \gamma \in \Gamma \text {. }
$$

Definition 6.8. A $(K / k, S)$-extension $\tilde{L} / k$ is universal if it satisfies the following:

(1) The Galois group $\tilde{H}=\operatorname{Gal}(\tilde{L} / K)$ is finite free over $\mathbb{Z}_{p}$.

(2) The induced pairing

$$
F_{p} \cdot U \times F_{p} \cdot X^{\prime}(K) \longrightarrow F_{p} \cdot I(\tilde{H}) / I(\tilde{H})^{2}=I_{F_{p}}(\tilde{H}) / I_{F_{p}}(\tilde{H})^{2}
$$

is universal.

Definition 6.9. An admissible extension $L / k$, as well as the Galois group $H=$ $\operatorname{Gal}(L / K)$, is said to be unrestricted if the following conditions are satisfied:

(1) $H \simeq \mathbb{Z}_{p}^{d}$, for some $d$.

(2) There exists a universal strict $(K / k, S)$-extension of $H$.

(3) The constant $\mathbb{Z}_{p}$-extension $L_{0} / K$ is a sub-extension of $L / K$.

Lemma 6.10. The following are true:

(1) Suppose that $\tilde{L}^{\prime} / k$ contains $\tilde{L} / k$ and they are both $(K / k, S)$-extensions. If $\tilde{H}^{\prime}:=\operatorname{Gal}\left(\tilde{L}^{\prime} / K\right)$ is finite free over $\mathbb{Z}_{p}$ and $\tilde{L} / k$ is universal, then $\tilde{L}^{\prime} / k$ is also universal.

(2) Suppose that $L^{\prime} / k$ contains $L / k$ and they are both admissible. If $H^{\prime}:=$ $\operatorname{Gal}\left(L^{\prime} / K\right)$ is finite free over $\mathbb{Z}_{p}$ and $L / k$ is unrestricted, then $L^{\prime} / k$ is also unrestricted.

(3) Every admissible extension whose Galois group is finitely generated over $\mathbb{Z}_{p}$ is contained in an unrestricted admissible extension.

Proof. Part (1) follows directly from the definitions. For part (2), we just note that if $\tilde{L} / k$ is a universal strict $(K / k, S)$-extension of $L / k$, then part (1) implies that $\tilde{L} L^{\prime} / K$ is a universal strict $(K / k, S)$-extension of $L^{\prime} / k$.

To prove part (3), we first note that by Lemma 3.3 of Tan95, a universal $(K / k, S)$-extension $M / k$ exists. We denote by $L^{\prime}$ the field obtained by adjoining $M$ with the given admissible extension and the constant $\mathbb{Z}_{p}$-extension. By Lemma 6.6. there is a $(K / k, S)$-extension $\tilde{L} / k$ such that $\tilde{L}$ contains $L^{\prime}$ and both $\operatorname{Gal}(\tilde{L} / K)$ and 
its maximal admissible quotient are finite free over $\mathbb{Z}_{p}$. Since $\tilde{L}$ contains $M$ and $M / k$ is universal, by part (1) $\tilde{L} / k$ is also universal. This completes the proof.

6.4. The decomposition. In view of (46), for the admissible Galois group $H$, the pairing $\langle\cdot, \cdot\rangle_{H, p}$ is $\Gamma$-invariant and hence cannot be universal. However, we are going to show that if $H$ is unrestricted, then its $\chi$-part, $\langle\cdot, \cdot\rangle_{\chi}$, is universal for every $\chi \in \hat{\Gamma}$.

Lemma 6.11. Assume that $H$ is unrestricted. The following are true:

(1) For each $\chi \in \hat{\Gamma}$, the pairing $\langle\cdot, \cdot\rangle_{\chi}$ is universal over $F_{p}$.

(2) The linear sub-spaces $\left\langle U_{\chi}, X_{\chi^{-1}}^{\prime}\right\rangle_{\chi}, \chi \in \hat{\Gamma}$, of $F_{p} \cdot I(H) / I(H)^{2}$ are linearly independent over $F_{p}$.

Proof. Let $\tilde{H}$ be a universal strict $(K / k, S)$-extension of $H$. Let $\tilde{H}^{(1)} \subset \tilde{H}$ be the subset of elements fixed under the action of $\Gamma$. Then the natural quotient map $\tilde{H} \longrightarrow H$ induces an isomorphism

$$
F_{p} \otimes_{\mathbb{Z}_{p}} \tilde{H}^{(1)} \longrightarrow F_{p} \otimes_{\mathbb{Z}_{p}} H .
$$

To simplify the notation, for the rest of the proof, for every Galois group $\mathcal{H}$, through the isomorphism $\delta_{\mathcal{H}}$ (see (13) $)$, we will identify $I(\mathcal{H}) / I(\mathcal{H})^{2}$ with $\mathcal{H}$.

By the $\Gamma$-equivariant property of the pairing $\langle\cdot, \cdot\rangle_{\tilde{H}}$, the restriction of $i d_{F_{p}} \otimes\langle\cdot, \cdot\rangle_{\tilde{H}}$ to $U_{\chi} \times X_{\chi^{-1}}^{\prime}$ has values all in $F_{p} \otimes_{\mathbb{Z}_{p}} \tilde{H}^{(1)}$.

To say that $i d_{F_{p}} \otimes\langle\cdot, \cdot\rangle_{\tilde{H}}$ is universal is the same as saying that the induced homomorphism $F_{p} \otimes U \otimes X^{\prime} \longrightarrow F_{p} \otimes_{\mathbb{Z}_{p}} \tilde{H}$ is an injection. Since $\bigoplus_{\chi \in \hat{\Gamma}} U_{\chi} \otimes X_{\chi^{-1}}^{\prime}$ is a direct summand of $F_{p} \otimes U \otimes X^{\prime}$ and its image under the induced homomorphism is in $F_{p} \otimes_{\mathbb{Z}_{p}} \tilde{H}^{(1)}$, the lemma is proved by taking the isomorphism (48).

6.5. The associated homogeneous polynomials. Let Ver denote the transfer homomorphism

$$
\text { Ver : } \begin{aligned}
G & \longrightarrow H, \\
g & \mapsto|\Gamma| g .
\end{aligned}
$$

For simplicity, we will also let Ver denote the restriction of it to a subgroup $H^{\prime}$ as well as the induced maps on augmentation quotients.

Definition 6.12. Suppose that $H \simeq \mathbb{Z}_{p}^{d}$ and $\mathcal{E}$ is a $\mathbb{Z}_{p}$ basis. Let $R=\mathcal{O}_{p}$ and recall the notation in (15), (16). For each $H^{\prime}$ define the homogeneous polynomial

$$
f_{H^{\prime}}:=d_{\mathcal{E}, F_{p}}\left(\left[\operatorname{Ver}\left(\operatorname{det}_{H^{\prime}}\right)\right]_{r_{K^{\prime}}}\right) \in F_{p}\left[s_{1}, \ldots, s_{d}\right] .
$$

Also, for each $\chi \in \hat{\Gamma}$, define the homogeneous polynomial

$$
f_{\chi}:=f_{\mathcal{E}, \chi}:=d_{\mathcal{E}, F_{p}}\left(\left[\operatorname{Ver}\left(\operatorname{det}_{\chi}\right)\right]_{r_{\chi}}\right) \in F_{p}\left[s_{1}, \ldots, s_{d}\right] .
$$

The polynomial $f_{H^{\prime}}$ is independent of the choice of the basis of $H$ up to elements of $\mathbb{Z}_{p}^{*}$. The polynomial $f_{\chi}$ is uniquely defined up to elements of $F_{p}^{*}$.

Definition 6.13. Let $H$ be an unrestricted admissible group. Then a $\mathbb{Z}_{p}$-basis $\mathcal{E}$ is called rational if the following conditions are satisfied:

$$
\left\langle U, X^{\prime}(K)\right\rangle_{H} \subset \sum_{\sigma \in \mathcal{E}} \mathbb{Z} \cdot \delta_{H}(\sigma)
$$


(2) If $L_{0} / K$ is the constant $\mathbb{Z}_{p}$-extension with $\operatorname{Gal}\left(L_{0} / K\right)=H_{0}=\mathbb{Z}_{p}$ and $\pi: H \longrightarrow H_{0}$ is the natural projection, then

$$
\pi(\sigma) \in \mathbb{Q} \cap \mathbb{Z}_{p} \subset \mathbb{Z}_{p}, \text { for all } \sigma \in \mathcal{E} .
$$

Lemma 6.14. An unrestricted admissible Galois group always has a rational basis.

Proof. Suppose that $H \simeq \mathbb{Z}_{p}^{d}$ is unrestricted. Let $A$ be a $\mathbb{Z}$-basis of $U$ and let $B$ be a $\mathbb{Z}$-basis of $X^{\prime}(K)$. We can find a $d$-dimensional $\mathbb{Q}$-vector space $V \subset \mathbb{Q}_{p} \otimes H$ such that $\left\{\langle a, b\rangle_{H} \mid a \in A, b \in B\right\} \subset \delta_{H}(V)$. Then $M:=V \cap H$ is a free $\mathbb{Z}$-module of rank $d$. Let $\mathcal{E}$ be a basis of $M$. Then obviously, the inclusion (50) holds. Lemma 6.3 implies that $\mathcal{E}$ is rational.

Proposition 6.15. Suppose that $H \simeq \mathbb{Z}_{p}^{d}$ is unrestricted and $\mathcal{E}$ is a rational basis. Then for each $H^{\prime}$, the homogeneous polynomial $f_{H^{\prime}}$ is in $\mathbb{Q}\left[s_{1}, \ldots, s_{d}\right]$, and for every $\chi$, the polynomial $f_{\chi}$ is absolutely irreducible and can be chosen in $F\left[s_{1}, \ldots, s_{d}\right]$ such that for some number $c \in F^{*}$

$$
f_{H^{\prime}}=c \cdot \prod_{\chi \in \hat{\Gamma}^{\prime}} f_{\chi} .
$$

Furthermore, the polynomials $\left\{f_{\chi} \mid \chi \in \hat{\Gamma}\right\}$ are algebraically independent over $F_{p}$.

Proof. The inclusion (50) implies that $f_{H} \in \mathbb{Q}\left[s_{1}, \ldots, s_{d}\right]$. Similarly, in $\sum_{u \in U} F \cdot u$,

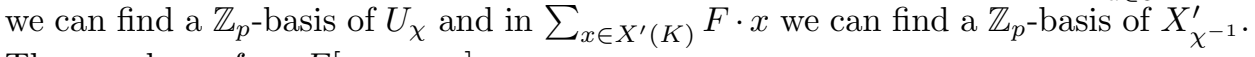
Then we have $f_{\chi} \in F\left[s_{1}, \ldots, s_{d}\right]$.

Since $H$ is fixed by $\Gamma$ and $\langle\cdot, \cdot\rangle_{H}$ is $\Gamma$-invariant, for $\chi^{\prime} \neq \chi^{-1}$ the restriction of $\langle\cdot, \cdot\rangle_{H, p}$ to the set $U_{\chi} \times X_{\left(\chi^{\prime}\right)^{-1}}^{\prime}$ is the trivial pairing. This shows the validity of (51) for $H^{\prime}=H$. In general, we use the compatibility equality (45) and view $\langle\cdot, \cdot\rangle_{H^{\prime}, p}$ as a part of $\langle\cdot, \cdot\rangle_{H, p}$.

For each integer $m$, consider the determinant of the $m$ by $m$ matrix $\left(t_{i j}\right)$, where $t_{i j}, i=1, \ldots, m, j=1, \ldots, m$, are independent variables over a field. It is well known that the determinant of this matrix is absolutely irreducible (see $\operatorname{Van} 70$ ). This fact and Lemma 6.11 imply the irreducibility and the algebraic independence of $f_{\chi}$.

6.6. The explicit expressions. Let $\eta$ and $w_{1}, \ldots, w_{n}$ be as in Definition 1.3 and let $w^{(i)} \in X^{\prime}(K)$ be such that $w^{(i)}\left(\sum_{w \in S(K)} \alpha_{w} w\right)=\alpha_{w_{i}}$, for every $\sum_{w \in S(K)} \alpha_{w} w \in$ $X^{\prime}(K)$. Then $\left\{w^{(1)}, \ldots, w^{(n)}\right\}$ generate a free $\mathbb{Z}[\Gamma]$-module of rank $n$. Assume that $\chi$ is a character of $\Gamma$ such that $r_{\chi}=n$. Then

$$
\left\{\frac{1}{|\Gamma|} \sum_{\gamma \in \Gamma} \chi(\gamma) \cdot \gamma w^{(i)} \mid i=1, \ldots, n\right\}
$$

is a basis of the $F$-vector space $X^{\prime}(K)_{\chi^{-1}}$. Also, if $M$ is a $\mathbb{Q}[\Gamma]$-free sub-space of $\mathbb{Q} \cdot U$ of rank $n$ and is generated over $\mathbb{Q}[\Gamma]$ by $\left\{\epsilon_{1}, \ldots, \epsilon_{n}\right\}$, then

$$
\left\{\sum_{\gamma \in \Gamma} \chi\left(\gamma^{-1}\right) \cdot \gamma \epsilon_{i} \mid i=1, \ldots, n\right\}
$$

is a basis of the $F_{p}$-vector space $U_{\chi}$. Using Definition 4.6 and equations (27) and (46), we obtain

$$
\begin{aligned}
{\left[\operatorname{Ver}(\operatorname{det})_{\chi}\right]_{r(\chi)} } & =\operatorname{Ver}\left(\operatorname{det}\left(\sum_{\gamma \in \Gamma} \chi(\gamma) \cdot \lambda_{\gamma\left(w_{j}\right)}\left(\epsilon_{i}\right)\right)_{\substack{i=1, \ldots, n \\
j=1, \ldots, n}}\right) \\
& =\operatorname{Ver}\left(\mathcal{R}_{\eta, \chi, H}^{\triangleright}\left(\epsilon_{1} \wedge \cdots \wedge \epsilon_{n}\right)\right) .
\end{aligned}
$$




\section{THE PROOF}

7.1. The product formula. As before, $H^{\prime}$ is a subgroup of $G$ such that $H \subset H^{\prime}$. Since $H^{\prime}$ is a closed subgroup, each character $\psi \in \hat{H}^{\prime}$ can be extended to a character on $G$. Let $\hat{\Gamma}^{\prime}{ }_{\psi}$ denote the set of all such extensions of $\psi$. Recall the definitions in Section 3.1 of the modified $L$-function $L_{S\left(K^{\prime}\right), T\left(K^{\prime}\right)}(\psi, s)$ and the Stickelberger element $\theta_{H^{\prime}}$. By the Class Field Theory, we have the following product formula:

$$
L_{S\left(K^{\prime}\right), T\left(K^{\prime}\right)}(\psi, s)=\prod_{\phi \in \hat{\Gamma}^{\prime} \psi} L_{S, T}(\phi, s) .
$$

Proposition 7.1. Suppose that $H^{\prime}$ is a subgroup of $G$ such that $H \subset H^{\prime}$. Then in the group ring $\mathcal{O}[G]$, we have

$$
\theta_{H^{\prime}}=\prod_{\chi \in \hat{\Gamma}^{\prime}} \theta_{\chi} .
$$

Proof. Apply every $\psi$ to both sides of (54); then use the product formula (53).

Definition 7.2. Assume that $H \simeq \mathbb{Z}_{p}^{d}$ and $\mathcal{E}=\left\{\sigma_{1}, \ldots, \sigma_{d}\right\}$ is a basis of $H$ over $\mathbb{Z}_{p}$. Let $R=\mathcal{O}_{p}$ and recall the notation in (15), (16)). For each $H^{\prime}$ define

$$
\xi_{H^{\prime}}=d_{\mathcal{E}, F_{p}}\left(\left[\operatorname{Ver}\left(\theta_{H^{\prime}}\right)\right]_{\left(r_{K^{\prime}}\right)}\right) \text {. }
$$

For each $\chi \in \hat{\Gamma}$, let $n_{\chi}$ be such that $\operatorname{Ver}\left(\theta_{\chi}\right) \in I_{\mathcal{O}_{p}}(H)^{n_{\chi}} \backslash I_{\mathcal{O}_{p}}(H)^{n_{\chi}+1}$ and define

$$
\xi_{\chi}=d_{\mathcal{E}, F_{p}}\left(\left[\operatorname{Ver}\left(\theta_{\chi}\right)\right]_{\left(n_{\chi}\right)}\right) .
$$

Proposition 7.3. Suppose that $H \simeq \mathbb{Z}_{p}^{d}$ is unrestricted and $\mathcal{E}$ is a rational basis. Then for each $H^{\prime}$, the homogeneous polynomial $\xi_{H^{\prime}}$ has all coefficients contained in $\mathbb{Q}$, and it satisfies

$$
\xi_{H^{\prime}}=h_{K^{\prime}, S\left(K^{\prime}\right), T\left(K^{\prime}\right)} f_{H^{\prime}}
$$

Furthermore, we have

$$
\xi_{H^{\prime}}=\prod_{\chi \in \hat{\Gamma}^{\prime}} \xi_{\chi} .
$$

Proof. It is a consequence of Theorem 5.3 and Proposition 7.1

7.2. The end of the proof. In this section, we complete the proof of the main theorem.

Proof. We only need to prove the theorem for the case where $H=\mathbb{H}$. Since $\mathbb{H}$ is the projective limit of unrestricted admissible groups, we will first consider the case where $H$ is unrestricted and $\mathcal{E}$ is a rational basis. For $\chi_{1}, \chi_{2} \in \hat{\Gamma}$, denote $\chi_{1} \sim \chi_{2}$ if they generate the same cyclic subgroup of $\hat{\Gamma}$.

Step 1. For each $\chi \in \hat{\Gamma}$, there is a character $\chi^{\prime} \sim \chi$ and a constant $c\left(\chi, \chi^{\prime}\right) \in F^{*}$ such that

$$
\xi_{\chi}=c\left(\chi, \chi^{\prime}\right) \cdot f_{\chi^{\prime}} .
$$

To show this, we recall equations (51), (55) and (56) and let $H^{\prime}$ run through all the subgroups of $G$ containing $H$. Consequently, there is a $c_{\chi} \in F_{p}^{*}$ such that

$$
\prod_{\chi^{\prime} \sim \chi} \xi_{\chi^{\prime}}=c_{\chi} \cdot \prod_{\chi^{\prime} \sim \chi} f_{\chi^{\prime}}
$$


Under the natural action of $\operatorname{Gal}(\overline{\mathbb{Q}} / \mathbb{Q})$ on $\hat{\Gamma}$, the set $\left\{\chi^{\prime} \mid \chi^{\prime} \sim \chi\right\}$ forms an orbit. For each $\tau \in \operatorname{Gal}(\overline{\mathbb{Q}} / \mathbb{Q})$, we have $\theta^{\tau} \chi={ }^{\tau} \theta_{\chi} \in F[G]$ and hence

$$
n_{\chi^{\prime}}=\operatorname{deg}\left(\xi_{\chi^{\prime}}\right)=\operatorname{deg}\left(\xi_{\chi}\right)=\operatorname{deg}\left(f_{\chi}\right)=r_{\chi}, \text { if } \chi^{\prime} \sim \chi .
$$

Since all the $f_{\chi^{\prime}}$ are absolutely irreducible and algebraically independent, there is a $\chi^{\prime}$ and a number $c\left(\chi, \chi^{\prime}\right)$ such that (57) holds.

We need to show $c\left(\chi, \chi^{\prime}\right) \in F^{*}$. The problem is that we don't know if the polynomial $\xi_{\chi}$ has its coefficients in $F$, although by Proposition 6.15 this holds for the polynomial $f_{\chi^{\prime}}$. To overcome this problem, we apply the ring homomorphism

$$
\pi_{*}: F_{p}[H] \longrightarrow F_{p}\left[H_{0}\right]
$$

induced from the natural projection

$$
\pi: H \longrightarrow H_{0}=\operatorname{Gal}\left(L_{0} / K\right)
$$

where, as before, $L_{0}$ is the constant $\mathbb{Z}_{p}$-extension of $K$. Let $\sigma$ be the Frobenius in $H_{0}$ and let $t=\sigma-1$. Then $\pi(\mathcal{E}) \subset \mathbb{Q} \sigma$ and $\pi_{*}\left(f_{\chi^{\prime}}\right)=b_{r_{\chi^{\prime}}} t^{r_{\chi}}$ with $b_{r_{\chi^{\prime}}} \in F$ for each $\chi^{\prime}$. Since $\pi_{*}\left(f_{H}\right)=b_{H} t^{r}$, where $b_{H}$ is a non-zero multiple of the classical regulator of $U$, by the product formula (51) we have $b_{r_{\chi^{\prime}}} \in F^{*}$.

Also, Lemma $3.2(2)$ and the functorial property of the Stickelberger elements imply that if $\pi_{*}\left(\operatorname{Ver}\left(\theta_{\chi}\right)\right)=a_{r_{\chi}} t^{r_{\chi}}+\cdots \in F_{p}[[t]]$, then $a_{r_{\chi}}$ is a non-zero rational multiple of the $r_{\chi}$ th derivative $L_{S, T}(\chi, 0)^{\left(r_{\chi}\right)} \in F^{*}$. Therefore

$$
c\left(\chi, \chi^{\prime}\right)=a_{r_{\chi}} / b_{r_{\chi}} \in F^{*} \text {. }
$$

Step 2. $\chi^{\prime}=\chi$.

To show this, we need to apply a work of Hayes. According to Hay88, our Theorem 5.1 and its consequence Theorem 5.4 are true in the case where $n=$ 1. Let $S^{(1)}=S \backslash\left\{v_{2}, \ldots, v_{n}\right\}$. Since $v_{1}, \ldots, v_{n} \in S$ split completely in $K$, the extension $K / k$ is unramified outside $S^{(1)}$. Also, $\# S^{(1)} \geq 2$, since $n \leq \# S-1$. If an abelian extension $L^{(1)} / K$ with Galois group $H^{(1)}$ is admissible with respect to the setting $\left(K / k, S^{(1)}\right)$, then it is also admissible with respect to $(K / k, S)$. We assume that $H^{(1)}$ is unrestricted with respect to $\left(K / k, S^{\prime}\right)$ and (after certain extension, if necessary) the given $L$ contains $L^{(1)}$. We will use $\theta^{(1)}, \xi^{(1)}, \operatorname{det}^{(1)}, f^{(1)}, \xi^{(1)}$ and so on to denote the objects derived from $H^{(1)}$ and $S^{(1)}$. Let $Q: H \longrightarrow H^{(1)}$ be the natural quotient map. Then for every $\psi \in \hat{\Gamma}$

$$
Q_{*}\left(\theta_{\psi}\right)=\left(1-\left[w_{2}\right]\right) \cdots\left(1-\left[w_{n}\right]\right) \cdot \theta_{\psi}^{(1)},
$$

where $\left[w_{i}\right] \in H^{\prime}$ is the Frobenius element at $w_{i}$. Also, there is a $c^{\prime}(\psi) \in F^{*}$ such that

$$
Q_{*}\left(\left[\operatorname{det}_{\psi}\right]_{\left(r_{\psi}\right)}\right)=c^{\prime}(\psi) \cdot\left(1-\left[w_{2}\right]\right) \cdots\left(1-\left[w_{n}\right]\right) \cdot\left[\operatorname{det}_{\psi}^{(1)}\right]_{\left(r_{\psi}-n+1\right)}
$$

Hayes' result together with equation (52) implies that

$$
Q_{*}\left(f_{\chi}\right)=c^{\prime \prime}(\chi) \cdot Q_{*}\left(\xi_{\chi}\right)
$$

for some $c^{\prime \prime}(\chi) \in F^{*}$. Equations (57) and (58) imply that $f_{\chi^{\prime}}^{(1)}$ and $f_{\chi}^{(1)}$ are proportional to each other. This cannot happen unless $\chi^{\prime}=\chi$, since $H^{(1)}$ is unrestricted and all the $f_{\psi}^{(1)}, \psi \in \hat{\Gamma}$, are algebraically independent.

Step 3. There is an $\epsilon$ in $\mathbb{Q} \Lambda^{n} U$ such that for every $\chi \in \hat{\Gamma}$,

$$
\left[\operatorname{Ver}\left(\theta_{\chi}\right)\right]_{(n)}=\operatorname{Ver}\left(\mathcal{R}_{\eta, \chi, H}^{\triangleright}(\epsilon)\right) .
$$


By (52), there is an $\epsilon^{\prime}$ in $\mathbb{Q} \Lambda^{n} U$ such that for each $\chi \in \hat{\Gamma}$ there is a $c_{\chi} \in F^{*}$ such that

Here we have

$$
\left[\operatorname{Ver}\left(\theta_{\chi}\right)\right]_{(n)}=c_{\chi} \cdot \operatorname{Ver}\left(\mathcal{R}_{\eta, \chi, H}^{\triangleright}\left(\epsilon^{\prime}\right)\right) \in I_{F_{p}}(H)^{n} / I_{F_{p}}(H)^{n+1} .
$$

$$
c_{\chi}=0, \text { for a character } \chi \text { such that } r_{\chi}>n,
$$

and also, for every $\tau \in \operatorname{Gal}(\overline{\mathbb{Q}} / \mathbb{Q})$,

$$
c^{c_{\chi}} \chi{ }^{\tau} c_{\chi} .
$$

Then there is an element $\alpha \in \mathbb{Q}[\Gamma]$ such that $\chi(\alpha)=c_{\chi}$ for every $\chi \in \hat{\Gamma}$. Let $\epsilon=\alpha \cdot \epsilon^{\prime}$. Then equation (59) holds for every $\chi$.

Step 4. $\theta_{G} \in I_{H}^{n}$.

It is easy to see that

$$
\text { Ver : } \begin{aligned}
I_{F_{p}}(H)^{m} / I_{F_{p}}(H)^{m+1} & \longrightarrow I_{F_{p}}(H)^{m} / I_{F_{p}}(H)^{m+1}, \\
x & \mapsto|\Gamma|^{m} x
\end{aligned}
$$

is injective.

As in Definition 3.3. for each $\gamma \in \Gamma$, let $\theta_{\gamma}$ be the $\gamma$-part of $\theta_{G}$. Then

$$
\operatorname{Ver}\left(\theta_{\chi}\right)=\sum_{\gamma \in \Gamma} \chi(\gamma) \cdot \operatorname{Ver}\left(\theta_{\gamma}\right)
$$

and equation (59) implies that each $\operatorname{Ver}\left(\theta_{\gamma}\right) \in I_{F_{p}}(H)^{n}$. If $\theta_{\gamma} \in I_{F_{p}, H}^{m} \backslash I_{F_{p}, H}^{m+1}$, then $£^{-1}\left(\left[\theta_{\gamma}\right]_{(m, H)}\right)=\gamma \otimes \theta_{[\gamma], m} \neq 0$, for some $\theta_{[\gamma], m} \in I_{F_{p}}(H)^{m} / I_{F_{p}}(H)^{m+1}$, and hence $\left[\operatorname{Ver}\left(\theta_{\gamma}\right)\right]_{(m, H)}=|\Gamma|^{m} \theta_{[\gamma], m} \neq 0$. But we also have $\left[\operatorname{Ver}\left(\theta_{\gamma}\right)\right]_{(m, H)}=\left[\operatorname{Ver}\left(\theta_{\gamma}\right)\right]_{(m)}$, which is zero unless $m \geq n$. This shows that every $\theta_{\gamma}$ is in $I_{F_{p}, H}^{n}$ and so is $\theta_{G}$. Lemma 2.9 and Lemma 2.10 imply that $\theta_{G} \in I_{H}^{n}$.

Step 5. $\left[\theta_{G}\right]_{(n, H)}=£\left(\mathcal{R}_{\eta, H}^{\triangleright}(\epsilon)\right)$.

Put

$$
\mathcal{R}_{\eta, H}^{\triangleright}(\epsilon)=\sum_{\gamma \in \Gamma} \gamma \otimes \mathcal{R}_{\gamma}, \quad \mathcal{R}_{\gamma} \in I_{F_{p}}(H)^{n} / I_{F_{p}}(H)^{n+1}
$$

and

$$
£^{-1}\left(\left[\theta_{G}\right]_{(n, H)}\right)=\sum_{\gamma \in \Gamma} \gamma \otimes \theta_{[\gamma]}, \quad \theta_{[\gamma]} \in I_{F_{p}}(H)^{n} / I_{F_{p}}(H)^{n+1} .
$$

Then

$$
\operatorname{Ver}\left(\mathcal{R}_{\eta, \chi, H}^{\triangleright}(\epsilon)\right)=\sum_{\gamma \in \Gamma}|\Gamma|^{n} \chi(\gamma) \mathcal{R}_{\gamma} \in I_{F_{p}}(H)^{n} / I_{F_{p}}(H)^{n+1}
$$

and

$$
\left[\operatorname{Ver} \theta_{\chi}\right]_{(n, H)}=\sum_{\gamma \in \Gamma}|\Gamma|^{n} \chi(\gamma) \theta_{[\gamma]} \in I_{F_{p}}(H)^{n} / I_{F_{p}}(H)^{n+1} .
$$

By applying the inverse Fourier transform to (59), we deduce that $\theta_{[\gamma]}=\mathcal{R}_{\gamma} \in$ $I_{F_{p}}(H)^{n}$ for every $\gamma \in \Gamma$, and hence

$$
\left[\theta_{G}\right]_{(n, H)}=£\left(\mathcal{R}_{\eta, H}^{\triangleright}(\epsilon)\right) .
$$

We have proved the above equality in the case where the coefficient ring is $F_{p}$, but Lemma 2.9 and Lemma 2.10 say that the same equality holds in the case where the coefficient ring is $\mathbb{Z}$.

Step 6. The case $H=\mathbb{H}$. 
Lemma 4.12 and Lemma 6.10 say that if $H$ is unrestricted and is large enough, then the exterior product $\epsilon$ in (62) is unique. Taking the projective limit, we see from the functorial properties that this $\epsilon$ also satisfies (62) for the case where $H=\mathbb{H}$. Corollary 4.11 says that $\epsilon \in \mathbb{Z}_{(p)} \Lambda_{0}^{n} U$. From (60), we see that $\epsilon \in \mathbb{Z}_{(p)} \Lambda_{S, T}^{n}$.

\section{REFERENCES}

[Aok91] N. Aoki, Gross' conjecture on the special values of abelian L-functions at $s=0$, Comm. Math. Univ. Sancti Pauli 40(1991) 101-124. MR1104783 (93f:11084)

[Aok03] N. Aoki, On Tate's refinement for a conjecture of Gross and its generalization, Journal de Theorie des Nombres Bordeaux 16 no. 3(2004) 457-486. MR2144953 (2006c:11134)

[Bun02] D. Burns, On relations between derivatives of abelian L-functions at $s=0$, preprint, 2002.

[Bun04] D. Burns, On the values of equivariant zeta functions of curves over finite fields, Documenta math. 9(2004) 357-399. MR2117419 (2005i:11079)

[Bun07] D. Burns, Congruences between derivatives of abelian L-functions at $s=0$, Invent. Math. 169(2007) 451-499. MR2336038

[BnL04] D. Burns and J. Lee, On the refined class number formula of Gross, J. Number Theory 107(2004) 282-286. MR2072389 (2005k:11227)

[Dar95] H. Darmon, Thaine's method for circular units and a conjecture of Gross, Canadian J. Math. 47(1995) 302-317. MR 1335080 (96h:11113)

[Gro88] B. Gross, On the values of abelian L-functions at $s=0$, J. Fac. Sci. Univ. Tokyo Sect. IA, Math. 35(1988) 177-197. MR931448 (89h:11071)

[Gro90] B. Gross, A note on the refined Stark Conjecture, unpublished.

[Hay88] D. Hayes, The refined p-adic abelian Stark conjecture in function fields, Invent. Math. 94(1988) 505-527. MR969242 (89k:11116)

[Haw04] A. Hayward, A class number formula for higher derivatives of abelian L-functions, Compositio Math. 140(2004) 99-129. MR1984423 (2004f:11133)

[Hua04] P.-Y. Huang, Gross' conjecture for extensions ramified over four points of $\mathbf{P}^{1}$, manuscript, 2004, submitted.

[Kis93] H. Kisilevsky, Multiplicative independence in function fields, J. Number Theory 44(1993) 352-355. MR1233295 (94k:11125)

[Lee97] J. Lee, On Gross' refined class number formula for elementary abelian extensions, J. Math. Sci. Univ. Tokyo 4(2)(1997) 373-383. MR1466351 (99c:11137)

[Lee02] J. Lee, Stickelberger elements for cyclic extensions and the order of vanishing of abelian $L$-functions at $s=0$, Compositio Math. 138(2003) 157-163. MR.2018824 (2004k:11177)

[Lee04] J. Lee, On the refined class number formula for global function fields, Math. Res. Letters 11(2004) 583-589. MR2106227 (2005k:11225)

[Pop99a] C.D. Popescu, Gras-type conjectures for function fields, Compositio Math. 118(1999), no. 3 263-290. MR1711315 (2000m:11116)

[Pop99b] C.D. Popescu, On a refined Stark conjecture for function fields, Compositio Math. 116(1999), no. 3 321-367. MR.1691163 (2000m:11115)

[Pop02] C.D. Popescu, Base change for Stark-type conjectures "over $\mathbb{Z}$ ", J. Reine Angew. Math. 542(2002), 85-111. MR 1880826 (2002k:11208)

[Pop05] C.D. Popescu, The Rubin-Stark conjecture for a special class of function field extensions, J. Number Theory 113(2005), no. 2 276-307. MR2153279(2006b:11143)

[Rei02] M. Reid, Gross' conjecture for extensions ramified over three points of $\mathbf{P}^{\perp}$, J. Math. Sci. Univ. Tokyo 10(2003), no. 1 119-138. MR.1963800 (2004a:11121)

[Rub96] K. Rubin, A Stark conjecture over $\mathbb{Z}$ for abelian L-functions with multiple zeros, Ann. Inst. Fourier 46(1996) 32-62. MR1385509 (97d:11174)

[San87] J. Sands, Stark's conjecture and abelian L-function with higher order zero at $s=0$, Advances in Math. 66(1987) 62-87. MR905927 (89g:11110)

[Stk71] H. M. Stark, L-functions at $s=1$. I. $L$-functions for quadratic forms, Adv. in Math. 7(1971) 301-343. MR0289429(44:6620)

[Stk75] H. M. Stark, L-functions at $s=1$. II. Artin $L$-functions with rational characters, Adv. in Math. 17(1975) 60-92-343. MR0382194(52:3082)

[Stk76] H. M. Stark, L-functions at $s=1$. III. Totally real fields and Hilbert's twelfth problem, Adv. in Math. 22(1976) 64-84. MR0437501 (55:10427) 
[Stk80] H. M. Stark, L-functions at $s=1$. IV. First derivatives at $s=0$, Adv. in Math. 35(1980) 197-235. MR563924 (81f:10054)

[Tat84] J. Tate, Les Conjectures de Stark sur les Fonctions L d'Artin en $s=0$ (Birkhauser, Boston, 1984). MR782485 (86e:11112)

[Tat97] J. Tate, A letter to Joongul Lee, 22 July 1997.

[Tat04] J. Tate, Refining Gross's conjecture on the values of abelian L-functions, Contemp. Math. 353(2004) 189-192. MR2088717 (2005g:11220)

[Tan95] K.-S. Tan, On the special values of abelian L-function, J. Math. Sci., Univ. Tokyo 1(1995) 305-319. MR1317462 (96b:11152)

[Tan04] K.-S. Tan, A note on Stickelberger elements for cyclic p-extensions over global function fields of characteristic p, Math. Res. Letters 11(2004) 273-278. MR2067472 (2005c:11142)

[Van70] B.L. Van Der Waerden, Algebra (Frederick Unger, New York, 1970).

[Yam89] M. Yamagishi, On a conjecture of Gross on special values of L-functions, Math. Z. 201(1989) 391-400. MR999736 (90g:11159)

Department of Mathematics, National Taiwan University, Taipei 10764, Taiwan

E-mail address: tan@math.ntu.edu.tw 